\title{
Nearby states in non-Hermitian quantum systems
}

\author{
Hichem Eleuch ${ }^{1 *}$ and Ingrid Rotter ${ }^{2 \dagger}$ \\ 1 Department of Physics, McGill University, Montreal, Canada H3A 2 T8 and \\ ${ }^{2}$ Max Planck Institute for the Physics of Complex Systems, D-01187 Dresden, Germany
}

(Dated: May 31, 2021)

\begin{abstract}
In part I, the formalism for the description of open quantum systems (that are embedded into a common well-defined environment) by means of a non-Hermitian Hamilton operator $\mathcal{H}$ is sketched. Eigenvalues and eigenfunctions are parametrically controlled. Using a $2 \times 2$ model, we study the eigenfunctions of $\mathcal{H}$ at and near to the singular exceptional points (EPs) at which two eigenvalues coalesce and the corresponding eigenfunctions differ from one another by only a phase. In part II, we provide the results of an analytical study for the eigenvalues of three crossing states. These crossing points are of measure zero. Then we show numerical results for the influence of a nearby ("third") state onto an EP. Since the wavefunctions of the two crossing states are mixed in a finite parameter range around an EP, three states of a physical system will never cross in one point. Instead, the wavefunctions of all three states are mixed in a finite parameter range in which the ranges of the influence of different EPs overlap. We may relate these results to dynamical phase transitions observed recently in different experimental studies. The states on both sides of the phase transition are non-analytically connected.
\end{abstract}

\footnotetext{
* email: heleuch@fulbrightmail.org

${ }^{\dagger}$ email: rotter@pks.mpg.de, author to whom correspondence should be addressed
} 


\title{
Part I: Two states
}

\begin{abstract}
The formalism for the description of open quantum systems (that are embedded into a common well-defined environment) by means of a non-Hermitian Hamilton operator $\mathcal{H}$ is sketched. Eigenvalues and eigenfunctions are parametrically controlled. Using a $2 \times 2$ model, we study the eigenfunctions of $\mathcal{H}$ at and near to the singular exceptional points (EPs) at which two eigenvalues coalesce and the corresponding eigenfunctions differ from one another by only a phase. Nonlinear terms in the Schrödinger equation appear nearby EPs which cause a mixing of the wavefunctions in a certain finite parameter range around the EP. The phases of the eigenfunctions jump by $\pi$ at an EP. These results hold true for systems that can emit ("loss") particles into the environment of scattering wavefunctions as well as for systems which can moreover absorb ("gain") particles from the environment. In a parameter range far from an EP, open quantum systems are described well by a Hermitian Hamilton operator. The transition from this parameter range to that near to an EP occurs smoothly.
\end{abstract}

\section{INTRODUCTION}

The basic features of quantum mechanics are worked out about 90 years ago: the Schrödinger equation is linear and allows superpositions of quantum states to be solutions of the Schrödinger equation; the Hamiltonian $H^{B}$ describing the system is Hermitian, its eigenvalues $E_{i}^{B}$ are real and its eigenfunctions $\Phi_{i}^{B}$ are normalized according to $\left\langle\Phi_{i}^{B} \mid \Phi_{j}^{B}\right\rangle=\delta_{i, j}$. The system described in this manner is closed since its coupling to an environment is not involved in the theory. The finite lifetime of most states of a (small) system is calculated by means of tunneling, without taking into account any feedback from the environment onto the system. This theory is proven experimentally during multi-year studies performed on different systems at low level density.

For the last years, not only the resolution of most experimental devices has increased considerably but also calculations with higher accuracy have become possible. As a result, the standard quantum theory has shown its limit to describe successfully experimental results. Counterintuitive results are obtained in different experiments. An example is the observation of an unexpected regularity of the measured transmission phases (so-called phase 
lapses) in mesoscopic systems [1] which could not explained in the framework of Hermitian quantum physics in spite of much effort [2, 3]. They are explainable however by considering the feedback from the environment onto the system [4]. Another example is the experimental observation and theoretical description of a dynamical phase transition (DPT) in the spin swapping operation [5, 6]. While Fermi's golden rule holds below the DPT, it is violated above it. In a new experimental paper [7], the formation of a protected sub-band for conduction in quantum point contacts under extreme biasing is found, see also [8]. This sub-band is a collective robust mode of non-equilibrium transport that is immune to local heating. It has potential practical implications for nanoscale devices made of quantum point contacts and quantum dots.

In order to improve the theoretical description, in some papers the coupling of the system to an environment is taken into account explicitly. Mostly, this is done by replacing the Hermitian Hamilton operator, or part of it, by a non-Hermitian one, see e.g. the reviews [9, 10] and the book [11]. In other papers, nonlinearities are added to the Schrödinger equation. An example is the review [12] where the role of nonlinear Fano resonances in theoretical and experimental studies of light propagation in photonic devices and charge transport through quantum dots (nanostructures) is reviewed. By this means, the description of experimental results could be improved considerably in all cases.

A non-Hermitian Hamiltonian in the Schrödinger equation appears when the system is considered to be open, i.e. to be embedded into an environment, and the coupling between the system and its environment is taken into account from the very beginning. A natural environment is the continuum of scattering wavefunctions to which the states of the system are coupled and into which they decay. It can be changed by external fields, however never be deleted. The finite lifetime of the states of the system is calculated directly from the non-Hermitian part of the Hamiltonian [9, 10]. The feedback from the environment onto the system is involved in the non-Hermitian Hamiltonian $\mathcal{H}$ and therefore also in its eigenvalues $\mathcal{E}_{i}$ and eigenfunctions $\Phi_{i}$. The basic assumption of this description is supported experimentally by the recent observation that remote states are coupled through the continuum [13].

Meanwhile there are many calculations performed with a non-Hermitian Hamiltonian. Usually, the behavior of the system is controlled by means of varying a certain parameter. The restriction of the parameter dependence of the Hamiltonian $\mathcal{H}$ to its explicitly non- 
Hermitian part (by neglecting the parameter dependence of its real Hermitian part) allows us to receive a quick overview on the spectroscopic redistribution processes occurring in the system under the influence of the coupling to the environment, see e.g. [4, 14, 15]. Most interesting is the appearance of unexpected collective coherent phenomena in different systems. They are similar to the phenomenon of Dicke superradiance [16] which is known in optics for many years. It has been shown, moreover, that the reorganization of the spectrum of the system under the influence of the coupling to the environment at a critical value of the control parameter, occurs globally over the whole energy range of the spectrum [14]. It takes place by a cooperative action of all states, and the length scale diverges as well as the degree of non-Hermiticity of the Hamiltonian. It has been shown further that the reordering of the spectrum corresponds, indeed, to a second-order phase transition [14], justifying the notation dynamical phase transition. The states below and beyond the DPT are non-analytically connected. This method is shown to describe also phase transitions in, e.g., biological systems [17].

The calculation of the eigenvalues $\mathcal{E}_{i}$ and eigenfunctions $\Phi_{i}$ of the non-Hermitian Hamiltonian $\mathcal{H}$ hits upon some mathematically non-trivial problems due to the existence of singular points in the continuum. At these points, two eigenvalues coalesce and the two corresponding eigenfunctions differ from one another only by a phase [10, 18]. The geometric phase of these points differs from the Berry phase of a diabolic point by a factor 2. These singular points, called usually exceptional points (EPs), are well-known in mathematics [19]. Their meaning for the dynamics of open quantum systems and the behavior of the two eigenfunctions at an EP is however studied only recently. Numerical results for the eigenvalues and eigenfunctions of $\mathcal{H}$ under the influence of an EP in a concrete system are obtained, e.g., for atoms [20, 21], for the transmission through quantum dots [22-25] and for charge transport in molecular networks [26]. In the early papers, the EPs are called mostly branch points in the complex plane or double poles of the $S$ matrix. Phase transitions in open quantum systems which are associated with the formation of long-lived and short-lived states according to [14], are related to EPs first in [27]. More recent results can be found in the review [10]. The drawback of all these studies is the unsolved question how different EPs influence one another and how they are related to a DPT.

The eigenfunctions of a symmetric non-Hermitian operator $\mathcal{H}$ are biorthogonal according to $\left\langle\Phi_{i}^{*}\right| \mathcal{H}=\mathcal{E}_{i}\left\langle\Phi_{i}^{*}\right|$ and $\mathcal{H}\left|\Phi_{i}\right\rangle=\mathcal{E}_{i}\left|\Phi_{i}\right\rangle$ (where $\mathcal{E}_{i}$ is a complex eigenvalue of $\mathcal{H}$ ). They have 
to be normalized therefore by means of $\left\langle\Phi_{i}^{*} \mid \Phi_{j}\right\rangle$ which is a complex number (in difference to the norm $\left\langle\Phi_{i} \mid \Phi_{j}\right\rangle$ which is a real number). In order to guarantee a smooth transition from the description of an open quantum system to an almost (and eventually really) closed one, the eigenfunctions of $\mathcal{H}$ should be normalized according to $\left\langle\Phi_{i}^{*} \mid \Phi_{j}\right\rangle=\delta_{i j}$. This is possible only by the additional requirement $\operatorname{Im}\left\langle\Phi_{i}^{*} \mid \Phi_{j}\right\rangle=0$. This condition implies that the relation between the phases of the two states $i$ and $j$ is, generally, not rigid: far from an EP, the two wavefunctions are (almost) orthogonal to one another in (nearly) the same manner as the eigenfunctions of a Hermitian operator while they become linearly dependent in approaching an EP [10] such that the biorthogonality of them cannot be neglected. This is quantitatively expressed by the phase rigidity $r_{i} \equiv\left\langle\Phi_{i}^{*} \mid \Phi_{j}\right\rangle /\left\langle\Phi_{i} \mid \Phi_{j}\right\rangle$ which is reduced in approaching an EP, $r_{i} \rightarrow 0$. Here, the environment can put its information into the system by aligning states of the system with states of the environment, i.e. by enhancing their decay width.

The phase rigidity of the eigenfunctions and its reduction near to the singular EP is the most interesting value when a realistic quantum system is described by a Schrödinger equation with non-Hermitian Hamiltonian. Since the environment is able to change the spectroscopic properties of the system only if $r_{i}<1$, an EP may influence strongly the dynamics of an open quantum system. This is in contrast to a closed system described by a Hermitian operator and rigid phases $\left(r_{i}=1\right)$ of its eigenfunctions. In [22, 23], the correlation between non-rigid phases of the eigenfunctions $\Phi_{i}$ of the non-Hermitian Hamiltonian in the neighborhood of an EP and the transmission through a quantum dot is demonstrated in calculations for a special quantum dot. The enhancement is a collective effect caused by $r_{i}<1$ for many levels $i$ in a certain finite parameter range. It has been shown further [10] that the Schrödinger equation of the system contains nonlinear terms when $r_{i}<1$, i.e. in the neighborhood of EPs. In contrast to the usual calculations, it is therefore not necessary to introduce nonlinear terms into the Schrödinger equation by hand. They are part and parcel of the non-Hermitian quantum physics, and appear only in the vicinity of EPs (where $\left.r_{i}<1\right)$.

Recently, non-Hermitian Hamiltonians are studied the eigenvalues of which are real in a broad parameter range [28]. Under certain conditions, the eigenvalues of the Hamiltonian become complex as shown theoretically [28] as well as experimentally [29 32]. The meaning of EPs for these processes is studied in different papers, e.g. [33 39]. Less studied is the question whether or not these processes can be considered to be a DPT in the sense 
described above. The main problem is similar to that appearing in the description of the Dicke superradiance, an effect known for many years [16], however not fully understood up to today. In both cases, the experimental studies are performed in optics. While the formal equivalence of the quantum mechanical Schrödinger equation and the optical wave equation in symmetric optical lattices [40-43] is explored in the first case for an interpretation of the experimental results, a comparable theoretical study does not exist in the second case, i.e. for the Dicke superradiance.

It is the aim of the present paper to study the meaning of the mathematical non-trivial properties of non-Hermitian operators for the physics of open quantum systems that are embedded into a common well-defined environment. The mathematical properties are the existence of singular points (EPs); the reduced phase rigidity $\left(r_{i}\right)$ in their vicinity; the appearance of nonlinear terms in the Schrödinger equation due to $r_{i}<1$; and the appearance of constructive interferences. The physical observable effects are DPTs known to appear at high level density. They will be discussed in the following paper [44].

In our calculations we use a schematic model to simulate typical features of open quantum systems that are induced coherently by the common environment. The obtained results are generic. The basic formalism used by us, is worked out in nuclear physics many years ago [45] where it is, however, used by introducing the non-Hermiticity by means of a perturbation and, furthermore, by using statistical assumptions for the individual states (mostly according to random matrix theory). In contrast to this, we consider directly the individual eigenvalues $\mathcal{E}_{i}$ and eigenfunctions $\Phi_{i}$ of the non-Hermitian Hamiltonian $\mathcal{H}$. In particular, we are interested in the influence of the EPs onto these values. As very well known, the eigenvalues show level repulsion and (or) width bifurcation. We show that the eigenfunctions contain new information, because they characterize the parameter range over which the influence of the EPs can be seen and the manner how different EPs may influence each another.

In the present paper, we consider a two-level system with real, complex and imaginary coupling coefficients between system and environment with loss (emission) of particles to the environment (Sect. III) which is the usual situation of quantum systems embedded into the environment of scattering wavefunctions. In Sect. III we consider systems in which additionally gain (absorption) of particles from the environment occurs what is discussed recently in literature, e.g. [36 39]. 
In a following paper [44], we address finally the problem of the relation between EPs and DPTs in systems with more than two nearby states coupled via a common environment. Here different EPs may influence each other.

\section{CROSSING OF TWO STATES IN AN OPEN QUANTUM SYSTEM WITH SYMMETRIC NON-HERMITIAN HAMILTONIAN}

\section{A. Basic equations, Hamiltonian near an exceptional point}

In an open quantum system, the discrete states described by a Hermitian Hamiltonian $H^{B}$, are embedded into the continuum of scattering wavefunctions, which exists always and can not be deleted. Due to this fact the discrete states turn into resonance states the lifetime of which is usually finite. The Hamiltonian $\mathcal{H}$ of the system which is embedded into the environment, is non-Hermitian. Its eigenvalues are complex and provide not only the energies of the states but also their lifetimes (being inverse proportional to the widths).

According to [45], the non-Hermitian Hamiltonian of an open quantum system reads [10]

$$
\mathcal{H}^{F}=H^{B}+V_{B C} G_{C}^{(+)} V_{C B}
$$

where the second term is the non-Hermitian perturbation; $V_{B C}$ and $V_{C B}$ stand for the inter-

action between system and environment; and $G_{C}^{(+)}$is the Green function in the environment. The so-called internal (first-order) interaction between two discrete states $i$ and $j$ is involved in $H^{B}$ while their external (second-order) interaction via the common environment is described by the last term of (11). Generally, the coupling matrix elements that determine the external interaction of two states consist of the principal value integral

$$
\operatorname{Re}\left\langle\Phi_{i}^{B}|\mathcal{H}| \Phi_{j}^{B}\right\rangle-E_{i}^{B} \delta_{i j}=\frac{1}{2 \pi} \mathcal{P} \int_{\epsilon_{c}}^{\epsilon_{c}^{\prime}} \mathrm{d} E^{\prime} \frac{\gamma_{i c}^{0} \gamma_{j c}^{0}}{E-E^{\prime}}
$$

which is real, and the residuum

$$
\operatorname{Im}\left\langle\Phi_{i}^{B}|\mathcal{H}| \Phi_{j}^{B}\right\rangle=-\frac{1}{2} \gamma_{i c}^{0} \gamma_{j c}^{0}
$$

which is imaginary [10]. Here, the $\Phi_{i}^{B}$ and $E_{i}^{B}$ are the eigenfunctions and (discrete) eigenvalues, respectively, of the Hermitian Hamiltonian $H^{B}$ which describes the states in the subspace of discrete states without any interaction of the states via the environment. The 
$\gamma_{i c}^{0} \equiv \sqrt{2 \pi}\left\langle\Phi_{i}^{B}|V| \xi_{c}^{E}\right\rangle$ are the (energy-dependent) coupling matrix elements between the discrete states $i$ of the system and the environment of scattering wavefunctions $\xi_{c}^{E}$. The $\gamma_{k c}^{0}$

have to be calculated for every state $i$ and for every channel $c$ (for details see [10]). When $i=j$, (22) and (3) give the selfenergy of the state $i$. The coupling matrix elements (22) and (3) (by adding $E_{i}^{B} \delta_{i j}$ in the first case) are often simulated by complex values $\omega_{i j}$, e.g. [46, 47].

In order to study the interaction of two states via the common environment it is best to start from a non-Hermitian Hamiltonian $\mathcal{H}$ in which $H^{B}$ in (11) is replaced by a nonHermitian Hamilton operator $\mathcal{H}_{0}$ the eigenvalues of which are complex (and not discrete as those of $H^{B}$ ). Let us consider, for example, the symmetric $2 \times 2$ matrix

$$
\mathcal{H}^{(2)}=\left(\begin{array}{cc}
\varepsilon_{1} \equiv e_{1}+\frac{i}{2} \gamma_{1} & \omega_{12} \\
\omega_{21} & \varepsilon_{2} \equiv e_{2}+\frac{i}{2} \gamma_{2}
\end{array}\right)
$$

with $\gamma_{i} \leq 0$. The diagonal elements of (4) are the two complex eigenvalues $\varepsilon_{i}(i=1,2)$ of the non-Hermitian operator $\mathcal{H}_{0}$. That means, the $e_{i}$ and $\gamma_{i}$ denote the energies and widths, respectively, of the two states when $\omega_{i j}=0$. The $\omega_{12}=\omega_{21} \equiv \omega$ stand for the coupling matrix elements of the two states via the common environment which are, generally, complex due to (21) and (3). The selfenergy of the states is assumed to be included into the $\varepsilon_{i}$. The Hamiltonian $\mathcal{H}^{(2)}$ allows us to consider the properties of the system near to and at an EP because here the distance between the two states, that coalesce at the EP, relative to one another is much smaller than that relative to the other states of the system. Note that the coupling matrix elements $\gamma_{k c}^{0}$ in (2) and (3) have the dimension of square root of energy while the widths $\gamma_{k}$ of the individual eigenstates in (4) have, of course, the dimension of energy.

\section{B. Eigenvalues of $\mathcal{H}^{(2)}$}

The eigenvalues of $\mathcal{H}^{(2)}$ are

$$
\mathcal{E}_{i, j} \equiv E_{i, j}+\frac{i}{2} \Gamma_{i, j}=\frac{\varepsilon_{1}+\varepsilon_{2}}{2} \pm Z ; \quad Z \equiv \frac{1}{2} \sqrt{\left(\varepsilon_{1}-\varepsilon_{2}\right)^{2}+4 \omega^{2}}
$$

where $E_{i}$ and $\Gamma_{i}$ stand for the energy and width, respectively, of the eigenstate $i$. When the energy detuning of the two levels is varied, different behaviors of the eigenvalues (5) will be observed which depend on the coupling strength $\omega$ between the states and their environment. Generally, resonance states with nonvanishing widths $\Gamma_{i}$ repel each other in 
energy according to $\operatorname{Re}(Z)$ while the widths bifurcate according to $\operatorname{Im}(Z)$. The transition from level repulsion to width bifurcation is studied numerically in e.g. [48]. The two states cross when $Z=0$. This crossing point is an EP according to the definition of Kato [19]. Here, the two eigenvalues coalesce, $\mathcal{E}_{1}=\mathcal{E}_{2}$.

According to (5), two interacting discrete states (with $\gamma_{1}=\gamma_{2}=0$ and $e_{1} \neq e_{2}$ ) avoid always crossing since $\omega$ and $\varepsilon_{1}-\varepsilon_{2}$ are real in this case and the condition $Z=0$ can not be fulfilled,

$$
\left(e_{1}-e_{2}\right)^{2}+4 \omega^{2}>0 .
$$

In this case, the EP can be found only by analytical continuation into the continuum. This situation is called usually avoided crossing of discrete states. It holds also for narrow resonance states if $Z=0$ cannot be fulfilled due to the small widths of the two states. The physical meaning of this result is very well known since many years: the avoided crossing of two discrete states at a certain critical parameter value [49, 50] means that the two states are exchanged at this point, including their populations (population transfer).

When $\omega=i \omega_{0}$ is imaginary,

$$
Z=\frac{1}{2} \sqrt{\left(e_{1}-e_{2}\right)^{2}-\frac{1}{4}\left(\gamma_{1}-\gamma_{2}\right)^{2}+i\left(e_{1}-e_{2}\right)\left(\gamma_{1}-\gamma_{2}\right)-4 \omega_{0}^{2}}
$$

is complex. The condition $Z=0$ can be fulfilled only when $\left(e_{1}-e_{2}\right)^{2}-\frac{1}{4}\left(\gamma_{1}-\gamma_{2}\right)^{2}=4 \omega_{0}^{2}$ and $\left(e_{1}-e_{2}\right)\left(\gamma_{1}-\gamma_{2}\right)=0$, i.e. when $\gamma_{1}=\gamma_{2}$ (while $\left.e_{1} \neq e_{2}\right)$. In this case

$$
\left(e_{1}-e_{2}\right)^{2}-4 \omega_{0}^{2}=0 \rightarrow e_{1}-e_{2}= \pm 2 \omega_{0}
$$

and two EPs appear. It holds further

$$
\begin{aligned}
& \left(e_{1}-e_{2}\right)^{2}>4 \omega_{0}^{2} \rightarrow Z \in \Re \\
& \left(e_{1}-e_{2}\right)^{2}<4 \omega_{0}^{2} \rightarrow Z \in \Im
\end{aligned}
$$

independent of the parameter dependence of the $e_{i}$. In the first case, the eigenvalues $\mathcal{E}_{i}=$ $E_{i}+i / 2 \Gamma_{i}$ differ from the original values $\varepsilon_{i}=e_{i}+i / 2 \gamma_{i}$ by a contribution to the energies and in the second case by a contribution to the widths. The width bifurcation starts in the very neighborhood of one of the EPs and becomes maximum in the middle between the two EPs. This happens at the crossing point $e_{1}=e_{2}$ where $\Delta \Gamma / 2 \equiv\left|\Gamma_{1} / 2-\Gamma_{2} / 2\right|=4 \omega_{0}$. A similar situation appears when $\gamma_{1} \approx \gamma_{2}$, see numerical results in Sect. IID, The physical meaning 
of this result is completely different from that discussed above for discrete and narrow resonance states. It means that different time scales may appear without any enhancement of the coupling strength to the continuum (for details see [51]).

The cross section can be calculated by means of the $S$ matrix $\sigma(E) \propto|1-S(E)|^{2}$. For an isolated resonance, it gives the well-known Breit-Wigner line shape according to

$$
S=1+i \frac{\Gamma_{1}}{E-E_{1}-\frac{i}{2} \Gamma_{1}}
$$

where $E$ is the energy and $E_{1}$ and $\Gamma_{1}$ are defined in Eq. (5). This expression can be rewritten as [52]

$$
S=\frac{E-E_{1}+\frac{i}{2} \Gamma_{1}}{E-E_{1}-\frac{i}{2} \Gamma_{1}}
$$

which is explicitly unitary when the energy dependence of the $E_{i}$ and $\Gamma_{i}$ is taken into account [10]. Extending the problem to that of two closely neighboring resonance states that are coupled to one common continuum of scattering wavefunctions the unitary representation (12) of the $S$ matrix reads (up to a background term) [10]

$$
S=\frac{\left(E-E_{1}+\frac{i}{2} \Gamma_{1}\right)\left(E-E_{2}+\frac{i}{2} \Gamma_{2}\right)}{\left(E-E_{1}-\frac{i}{2} \Gamma_{1}\right)\left(E-E_{2}-\frac{i}{2} \Gamma_{2}\right)} .
$$

In this expression, the influence of an EP onto the cross section is contained in the eigenvalues $\mathcal{E}_{i}=E_{i}+i / 2 \Gamma_{i}$ of $\mathcal{H}^{(2)}$. Reliable results can be obtained therefore also when an EP is approached and the $S$ matrix has a double pole at the parameter value corresponding to the EP. Here, the line shape of the two overlapping resonances is described by

$$
S=1+2 i \frac{\Gamma_{d}}{E-E_{d}-\frac{i}{2} \Gamma_{d}}-\frac{\Gamma_{d}^{2}}{\left(E-E_{d}-\frac{i}{2} \Gamma_{d}\right)^{2}}
$$

by rewriting (13), where $E_{1}=E_{2} \equiv E_{d}$ and $\Gamma_{1}=\Gamma_{2} \equiv \Gamma_{d}$. It deviates from the Breit-Wigner line shape of an isolated resonance due to interferences between the two resonances. The first term of (14) is linear (with the factor 2 in front) while the second one is quadratic. As a result, two peaks with asymmetric line shape appear in the cross section (for a numerical example see Fig. 9 in [53]).

\section{Eigenfunctions of $\mathcal{H}^{(2)}$}

The eigenfunctions of a non-Hermitian $\mathcal{H}$ must fulfill the conditions $\mathcal{H}\left|\Phi_{i}\right\rangle=\mathcal{E}_{i}\left|\Phi_{i}\right\rangle$ and $\left\langle\Psi_{i}\right| \mathcal{H}=\mathcal{E}_{i}\left\langle\Psi_{i}\right|$ where $\mathcal{E}_{i}$ is an eigenvalue of $\mathcal{H}$ and the vectors $\left|\Phi_{i}\right\rangle$ and $\left\langle\Psi_{i}\right|$ denote its right 
and left eigenfunctions, respectively. When $\mathcal{H}$ is a Hermitian operator, the $\mathcal{E}_{i}$ are real, and we arrive at the well-known relation $\left\langle\Psi_{i}\right|=\left\langle\Phi_{i}\right|$. In this case, the eigenfunctions can be normalized by using the expression $\left\langle\Phi_{i} \mid \Phi_{j}\right\rangle$. For the symmetric non-Hermitian Hamiltonian $\mathcal{H}^{(2)}$, however, we have $\left\langle\Psi_{i}\right|=\left\langle\Phi_{i}^{*}\right|$. This means, the eigenfunctions are biorthogonal and have to be normalized by means of $\left\langle\Phi_{i}^{*} \mid \Phi_{j}\right\rangle$. This is, generally, a complex value, in contrast to the real value $\left\langle\Phi_{i} \mid \Phi_{j}\right\rangle$ of the Hermitian case. To smoothly describe the transition from a closed system with discrete states, to a weakly open one with narrow resonance states, we normalize the $\Phi_{i}$ according to

$$
\left\langle\Phi_{i}^{*} \mid \Phi_{j}\right\rangle=\delta_{i j}
$$

(for details see sections 2.2 and 2.3 of [10]). It follows

$$
\left\langle\Phi_{i} \mid \Phi_{i}\right\rangle=\operatorname{Re}\left(\left\langle\Phi_{i} \mid \Phi_{i}\right\rangle\right) ; \quad A_{i} \equiv\left\langle\Phi_{i} \mid \Phi_{i}\right\rangle \geq 1
$$

and

$$
\begin{gathered}
\left\langle\Phi_{i} \mid \Phi_{j \neq i}\right\rangle=i \operatorname{Im}\left(\left\langle\Phi_{i} \mid \Phi_{j \neq i}\right\rangle\right)=-\left\langle\Phi_{j \neq i} \mid \Phi_{i}\right\rangle \\
\left|B_{i}^{j}\right| \equiv \mid\left\langle\Phi_{i}\left|\Phi_{j \neq i}\right| \geq 0\right.
\end{gathered}
$$

At an EP $A_{i} \rightarrow \infty$ and $\left|B_{i}^{j}\right| \rightarrow \infty$. The $\Phi_{i}$ contain (like the $\mathcal{E}_{i}$ ) global features that are caused by many-body forces induced by the coupling $\omega_{i k}$ of the states $i$ and $k \neq i$ via the environment (which has an infinite number of degrees of freedom). The eigenvalues $\mathcal{E}_{i}$ and eigenfunctions $\Phi_{i}$ contain moreover the self-energy contributions of the states $i$ due to their coupling to the environment.

At the EP, the eigenfunctions $\Phi_{i}^{\mathrm{cr}}$ of $\mathcal{H}^{(2)}$ of the two crossing states differ from one another only by a phase,

$$
\Phi_{1}^{\mathrm{cr}} \rightarrow \pm i \Phi_{2}^{\mathrm{cr}} ; \quad \Phi_{2}^{\mathrm{cr}} \rightarrow \mp i \Phi_{1}^{\mathrm{cr}}
$$

according to analytical as well as numerical and experimental studies, see Appendix of [51], section 2.5 of [10] and Figs. 4 and 5 in [35]. That means, the wavefunction $\Phi_{1}$ of the state 1 jumps, at the EP, via the wavefunction $\Phi_{1} \pm i \Phi_{2}$ of a chiral state to $\pm i \Phi_{2}$ [54].

The Schrödinger equation with the non-Hermitian operator $\mathcal{H}^{(2)}$ is equivalent to a Schrödinger equation with $\mathcal{H}_{0}$ and source term [55]

$$
\left(\mathcal{H}_{0}-\varepsilon_{i}\right)\left|\Phi_{i}\right\rangle=-\left(\begin{array}{cc}
0 & \omega_{i j} \\
\omega_{j i} & 0
\end{array}\right)\left|\Phi_{j}\right\rangle \equiv W\left|\Phi_{j}\right\rangle .
$$


Due to the source term, two states are coupled via the common environment of scattering wavefunctions into which the system is embedded, $\omega_{i j}=\omega_{j i} \equiv \omega$. The Schrödinger equation (19) with source term can be rewritten in the following manner [55],

$$
\left(\mathcal{H}_{0}-\varepsilon_{i}\right)\left|\Phi_{i}\right\rangle=\sum_{k=1,2}\left\langle\Phi_{k}|W| \Phi_{i}\right\rangle \sum_{m=1,2}\left\langle\Phi_{k} \mid \Phi_{m}\right\rangle\left|\Phi_{m}\right\rangle .
$$

According to the biorthogonality relations (16) and (17) of the eigenfunctions of $\mathcal{H}^{(2)}$, (20) is a nonlinear equation. Most important part of the nonlinear contributions is contained in

$$
\left(\mathcal{H}_{0}-\varepsilon_{n}\right)\left|\Phi_{n}\right\rangle=\left\langle\Phi_{n}|W| \Phi_{n}\right\rangle\left|\Phi_{n}\right|^{2}\left|\Phi_{n}\right\rangle .
$$

The nonlinear source term vanishes far from an EP where $\left\langle\Phi_{k} \mid \Phi_{k}\right\rangle \rightarrow 1$ and $\left\langle\Phi_{k} \mid \Phi_{l \neq k}\right\rangle=$ $-\left\langle\Phi_{l \neq k} \mid \Phi_{k}\right\rangle \rightarrow 0$ as follows from the normalization (15). Thus, the Schrödinger equation with source term is linear far from an EP, as usually assumed. It is however nonlinear in the neighborhood of an EP.

The biorthogonality of the eigenfunctions $\Phi_{k}$ of the non-Hermitian operator $\mathcal{H}^{(2)}$ is determined quantitatively by the ratio

$$
r_{k} \equiv \frac{\left\langle\Phi_{k}^{*} \mid \Phi_{k}\right\rangle}{\left\langle\Phi_{k} \mid \Phi_{k}\right\rangle}=A_{k}^{-1} .
$$

Usually $r_{k} \approx 1$ for decaying states which are well separated from other decaying states (according to the fact that Hermitian quantum physics is a good approach at low level density). The situation changes however completely when an EP is approached:

(i) When two levels are distant from one another, their eigenfunctions are (almost) orthogonal, $\left\langle\Phi_{k}^{*} \mid \Phi_{k}\right\rangle \approx\left\langle\Phi_{k} \mid \Phi_{k}\right\rangle \equiv A_{k} \approx 1$.

(ii) When two levels cross at the EP, their eigenfunctions are linearly dependent according to (18) and $\left\langle\Phi_{k} \mid \Phi_{k}\right\rangle \equiv A_{k} \rightarrow \infty$.

These two relations show that the phases of the two eigenfunctions relative to one another change dramatically when the crossing point (EP) is approached. We call $r_{k}$, defined by (22), the phase rigidity of the eigenfunction $\Phi_{k}$. Generally $1 \geq r_{k} \geq 0$. The nonrigidity $r_{k}$ of the phases of the eigenfunctions of $\mathcal{H}^{(2)}$ follows directly from the fact that $\left\langle\Phi_{k}^{*} \mid \Phi_{k}\right\rangle$ is a complex number (in difference to the norm $\left\langle\Phi_{k} \mid \Phi_{k}\right\rangle$ which is a real number) such that the normalization condition (15) can be fulfilled only by the additional postulation $\operatorname{Im}\left\langle\Phi_{k}^{*} \mid \Phi_{k}\right\rangle=0$ (what corresponds to a rotation). 
When $r_{k}<1$, an analytical expression for the eigenfunctions as function of a certain control parameter can, generally, not be obtained. The non-rigidity $r_{k}<1$ of the phases of the eigenfunctions of $\mathcal{H}^{(2)}$ in the neighborhood of EPs is the most important difference between the non-Hermitian quantum physics and the Hermitian one. It expresses the fact that two nearby states can strongly interact with one another, when their wavefunctions are not supposed to be everywhere orthogonal (as in Hermitian quantum physics). Mathematically, $r_{k}<1$ causes nonlinear effects in quantum systems in a natural manner, as shown above. Physically, it gives the possibility that one of the states of the system aligns at (or near to) the EP with the common environment and receives, by this, a large width. This alignment is nothing but a quantitative measure of the influence of the environment onto the spectroscopic properties of the system [10].

It is meaningful to represent the eigenfunctions $\Phi_{i}$ of $\mathcal{H}^{(2)}$ in the set of basic wavefunctions $\Phi_{i}^{0}$ of $\mathcal{H}_{0}$

$$
\Phi_{i}=\sum_{j=1}^{N} b_{i j} \Phi_{j}^{0} \quad ; \quad b_{i j}=\left|b_{i j}\right| e^{i \theta_{i j}} .
$$

Also the $b_{i j}$ are normalized according to the biorthogonality relations of the wavefunctions $\left\{\Phi_{i}\right\}$. The angle $\theta_{i j}$ can be determined from $\operatorname{tg}\left(\theta_{i j}\right)=\operatorname{Im}\left(b_{i j}\right) / \operatorname{Re}\left(b_{i j}\right)$.

It should be mentioned here that the eigenfunctions $\Phi_{k}$ of $\mathcal{H}^{(2)}$ represent only the part of the resonance wavefunction that is localized inside the system. The wavefunction of the resonance state $k$ in the whole function space of discrete and scattering states contains additionally a "tail" due to its coupling to the scattering wavefunctions, see [10].

\section{Numerical results}

In our calculations, the mixing coefficients $b_{i j}$, defined in (23), of the wavefunctions of the two states are calculated by taking into account the fact that the mixing depends on the distance (in energy) of the two states, what can be simulated by assuming a Gaussian distribution

$$
\omega_{i \neq j}=\omega e^{-\left(e_{i}-e_{j}\right)^{2}}
$$

for the coupling coefficients. The results reproduce very well [46, 47] those obtained numer-

ically exact in [55] for two levels and real coupling $\omega$. Further, the selfenergies of the states are assumed, in our calculations, to be included into the $\varepsilon_{i}$. 

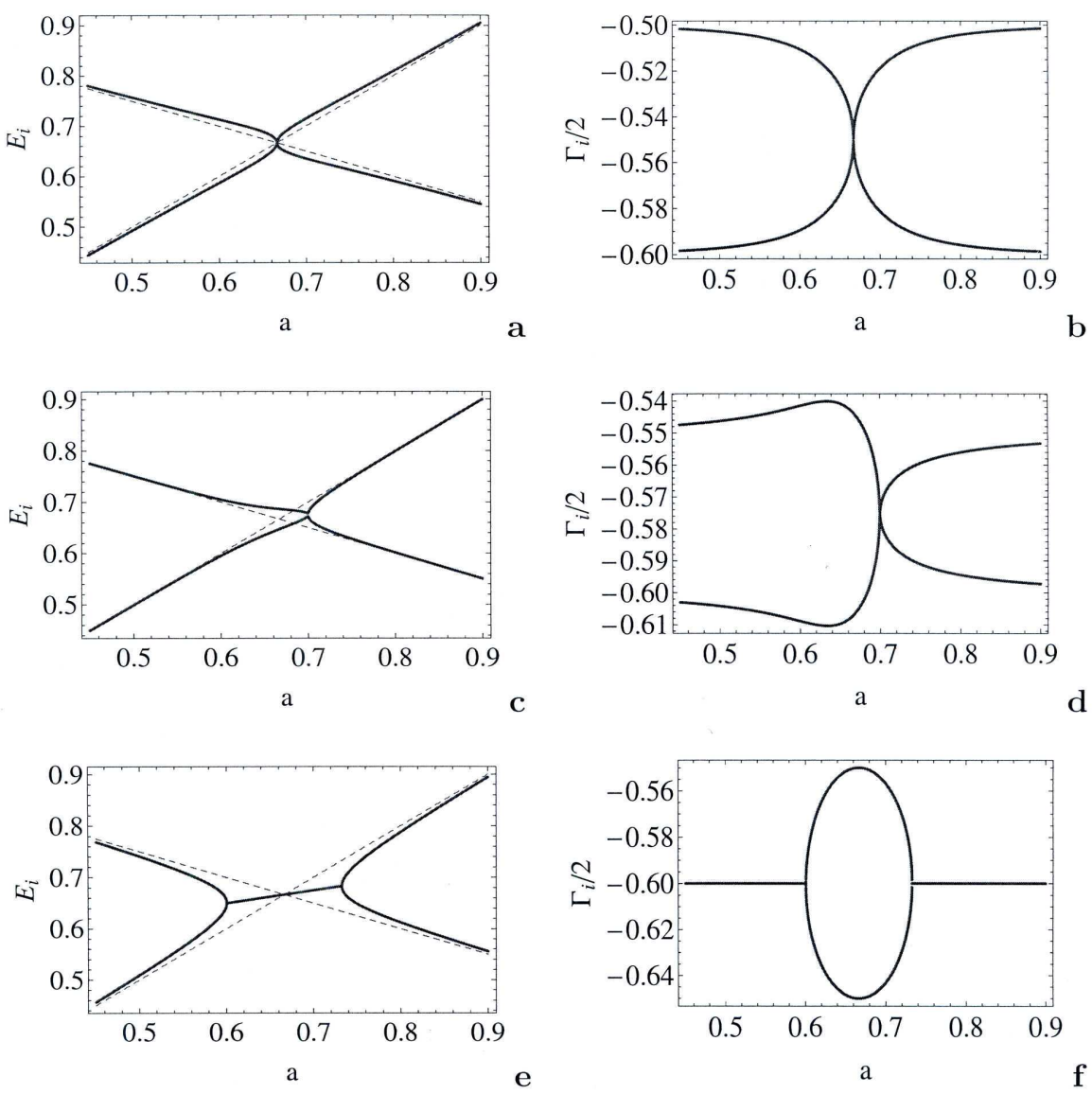

FIG. 1: Energies $E_{i}$ (left panel) and widths $\Gamma_{i} / 2$ (right panel) of $N=2$ states coupled to a common channel as a function of $a$. Parameters: $e_{1}=1-0.5 a ; \quad e_{2}=a ; \quad \gamma_{1} / 2=-0.5(\mathrm{a}, \mathrm{b}) ; \quad-0.5505(\mathrm{c}, \mathrm{d}) ; \quad-0.6(\mathrm{e}, \mathrm{f})$; $\gamma_{2} / 2=-0.6 ; \quad \omega=0.05(\mathrm{a}, \mathrm{b}) ; 0.025(1+\mathrm{i})(\mathrm{c}, \mathrm{d}) ; 0.05 i(\mathrm{e}, \mathrm{f})$. The dashed lines in $(\mathrm{a}, \mathrm{c}, \mathrm{e})$ show $e_{i}(a)$.

Let us first consider the $2 \times 2$ matrix (4) with $e_{1}=1-\frac{a}{2} ; e_{2}=a$ and with $\gamma_{i}(i=1,2)$ and $\omega_{12}=\omega_{21} \equiv \omega$ independent of $a$. For illustration, we show in Fig. 1 the eigenvalue trajectories $E_{i}(a)$ and $\Gamma_{i} / 2(a)$ and in Fig. 2 the mixing coefficients $b_{i j}=\left|b_{i j}\right| e^{i \theta_{i j}}$ (defined in (23) ) of the eigenfunctions of $\mathcal{H}^{(2)}$ as a function of $a$ in the neighborhood of an EP. The calculations are performed with real, complex and imaginary coupling coefficients $\omega$. Both, the upper (real $\omega$ ) and middle (complex $\omega$ ) rows of Figs. 1 and 2 show an EP at the critical parameter value $a=a_{\mathrm{cr}}$. Here the eigenvalue trajectories cross and $\left|b_{i j}\right| \rightarrow \infty$. The lower row is calculated with imaginary $\omega$ and $\gamma_{1}=\gamma_{2}$. Here two EPs appear, and $\left|b_{i j}\right| \rightarrow \infty$ at every EP.

The main difference of the eigenvalue trajectories with real to those with imaginary coupling coefficients $\omega$ are related to the relations (6) to (10) obtained analytically and 

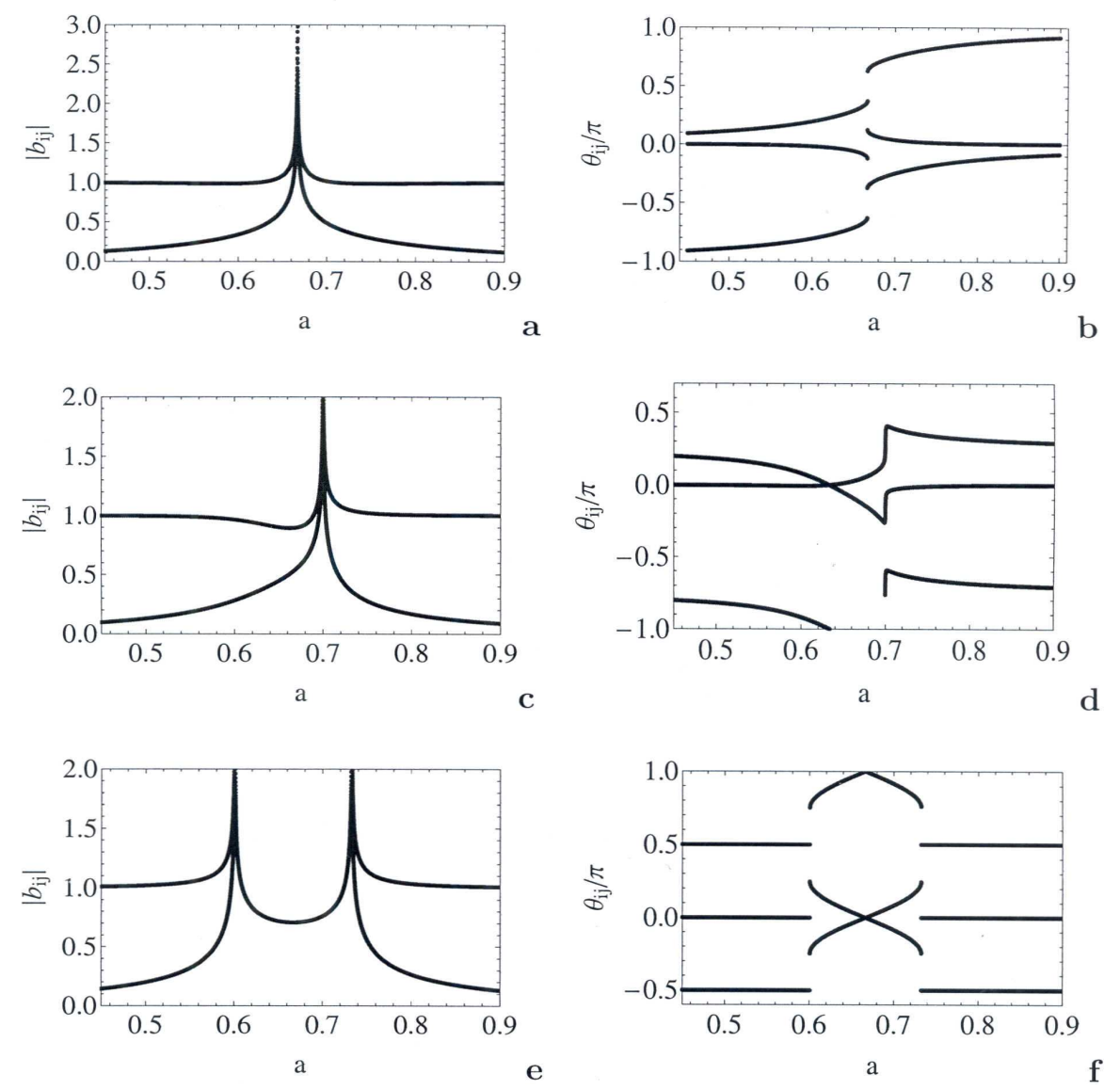

FIG. 2: Mixing coefficients $b_{i j}=\left|b_{i j}\right| e^{i \theta_{i j}}$ of $N=2$ states coupled to a common channel as a function of $a$. The parameters are the same as in Fig. 1,

discussed in Sect. IIB, For real and complex $\omega$ and $\gamma_{1} \neq \gamma_{2}$, the results show one EP (when the condition $Z=0$ is fulfilled, see Fig. [1, upper and middle rows). This EP is isolated from other EPs, generally. In the case of imaginary $\omega$ and $\gamma_{1} \approx \gamma_{2}$, however, two related EPs appear (Fig. 1, lower row). Between these two EPs, the widths $\Gamma_{i}$ bifurcate: the width of one of the two states increases by varying $a$ although the coupling strength $\omega$ between system and environment remains constant.

As can be seen from Fig. 2 left panel, the critical parameter range has a finite extension at both sides of the EPs. When $\omega$ is imaginary, the critical parameter range includes both EPs and their vicinity. Between the two EPs the eigenfunctions are strongly mixed (1:1) with one another. Beyond the critical parameter region, the eigenvalues trajectories $\mathcal{E}_{i}(a)$ approach the trajectories $\varepsilon_{j}(a)$ after exchange of $i$ and $j$.

Interesting are also the phases of the eigenfunctions in the neighborhood of an EP, see 
Fig. 2 right panel. The phases of all components of the eigenfunctions jump at the EP either by $-\pi / 4$ or by $+\pi / 4$. That means the phases of both eigenfunctions jump in the same direction by the same amount. Thus, there is a phase jump of $-\pi / 2$ (or $+\pi / 2)$ when one of the eigenfunctions passes into the other one at the EP. This result is in agreement with (18). It holds true for real as well as for complex and imaginary $\omega$ as can be seen from Fig. 2 right panel.

The position of an isolated EP can always be found by varying another parameter. For example, with $e_{1}=1-\frac{a}{2}+r \cos \theta ; e_{2}=a+r \sin \theta$ one EP appears in any case in the parameter range $0 \leq \theta \leq \pi$. The results obtained in the neighborhood of and at this EP show the same characteristic features as those in Figs. 11 and 2, around the crossing point (EP) of the eigenvalue trajectories, the eigenfunctions are mixed and $\left|b_{i j}\right| \rightarrow \infty$ at the EP. The phase jumps are of the same type as those shown in Fig. 2, confirming the relation (18) between the two eigenfunctions at the EP also by these calculations.

Now we explore numerically the phase difference $\Omega$ between the two eigenfunctions of the operator $\mathcal{H}^{(2)}$ that describes a 2-level open quantum system. The calculations are performed by starting from the unperturbed energies $\varepsilon_{k}=e_{k}+\frac{1}{2} \gamma_{k}$ (diagonal matrix elements of (4)), with the assumptions that $e_{1}=$ const while $e_{2}=e_{2}(d)$ depends on the distance $d$ between the two states which cross at $d=0$. The widths of both states are assumed to be constant, $\gamma_{k}=$ const for $k=1,2$. The angle $\Omega$ between the two eigenvectors of $\mathcal{H}^{(2)}$ is represented in the figures by $\cos (\Omega)$ in order to illustrate the changes of $\Omega$ in approaching an EP. The coupling strength $\omega$ is chosen to be real (Fig. 3), complex (Fig. 4left panel), and imaginary (Fig. 4 right panel). In the first and second case, we have one EP while in the last case, there are two EPs according to (8).

The results shown in Figs. 3 and 4 are the following. (i) For distant levels, the two eigenfunctions are almost orthogonal. Here, asymptotically $\cos (\Omega) \approx 0$, however the value $\cos (\Omega)$ never vanishes (see Fig. 3. d in logarithmic scale). (ii) At the EP, the eigenfunctions are linearly dependent from one another according to (18), what is expressed by $\cos (\Omega) \rightarrow$ \pm 1 in approaching the EP. These results confirm the statements according to which the normalization of the eigenfunctions of a non-Hermitian operator by means of the complex value (15) is possible only by rotating the eigenvector such that $\operatorname{Im}\left\langle\Phi_{k}^{*} \mid \Phi_{k}\right\rangle=0$. The rotation angle, represented by $\cos (\Omega)$, is shown in Figs. 3. $\mathrm{c}$, and $4 . \mathrm{c}$ and $\mathrm{f}$ for different values of the coupling coefficient $\omega$. 
As can be seen from the eigenvalue equations (5) and from Figs. 11 to 4, two states may avoid crossing at the EP by level repulsion (as very well known since many years [49, 50]), or they may cross freely while their widths bifurcate. In the last case, the lifetimes of the two states may finally differ strongly from one another, even bound states in the continuum may arise. The existence of these states is discussed already in the very early days of quantum mechanics [56], later considered in atomic physics [57, 58] and other systems, e.g. [25]. The eigenvalues of the Hamiltonian show the existence and position of the critical parameter values (corresponding to the EPs) at which level repulsion or width bifurcation takes place.

Figs. 1 to 4 illustrate furthermore how an EP influences its neighborhood and determines the dynamics of an open quantum system. (i) The wavefunctions of the two crossing states are mixed and the phases of the wavefunctions of the two states relative to one another vary in a finite parameter range in the neighborhood of the EP. The reduction of the phase rigidity $r_{k}$ (corresponding to (22) ) allows one of the states to align to the states of the environment, i.e. to receive a large width, while the other state almost decouples from the environment. (ii) When the interaction of the two states via the environment is imaginary and the widths of both states are similar to one another $\left(\gamma_{1} \approx \gamma_{2}\right)$, width bifurcation occurs between the two EPs according to (8) and (10) without any enhancement of the coupling strength to the environment. The phases jump at the two EPs in different directions and the eigenvalues approach the original values only beyond the two EPs.

Figs. 1 to 4 illustrate the most important difference between Hermitian and nonHermitian quantum physics: the phases of the eigenfunctions of a Hermitian operator relative to one another are fixed by the orthogonality relations at all parameter values, while those of $\mathcal{H}^{(2)}$ are not everywhere rigid. They are influenced by the singular points (EPs) at which two eigenvalues of the non-Hermitian operator $\mathcal{H}$ coalesce. Here, the two eigenstates are exchanged, what is accompanied by a change of the angle between the two eigenvectors according to (18). This process occurs not only at the position of the EP but is characteristic for a certain finite parameter range around it, as can be seen from the numerical results for the phase rigidity $r_{k}$ and for the angle $\Omega$ between the two eigenvectors.

Of prime importance for physical processes induced by an EP in an open quantum system that is embedded into a common well-defined environment, are the nonlinear terms occurring in the Schrödinger equation in the whole function space where $r_{k}<1$, see Eqs. (20) and (21). Eventually, they allow for some stabilization of the system by putting information 

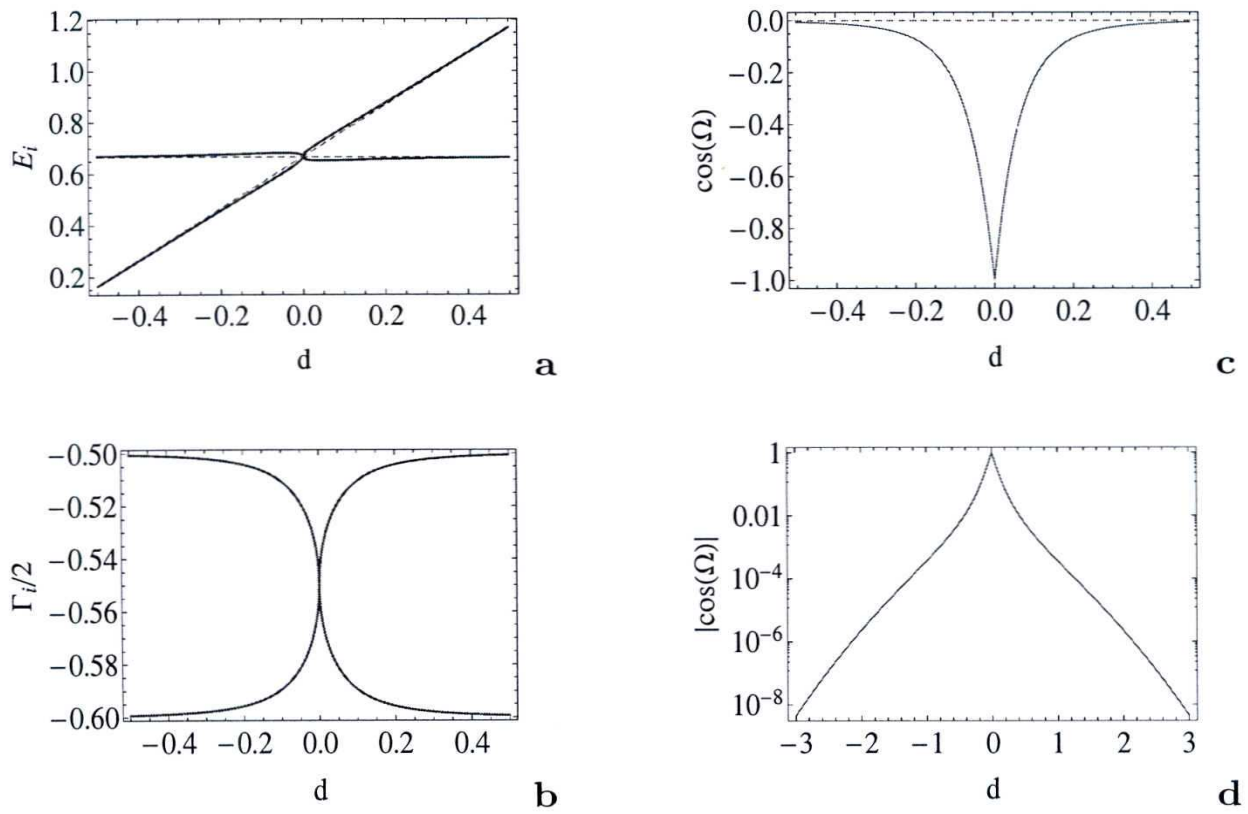

FIG. 3: Energies $E_{i}$ (full lines) (a), widths $\Gamma_{i} / 2(\mathrm{~b})$, and $\cos (\Omega),(\mathrm{c})$ in linear scale, (d) in logscale, as function of the distance $d$ for $N=2$ states coupled to one channel. The unperturbed energies are $e_{1}=2 / 3$ and $e_{2}=2 / 3+d$ (dashed lines in (a)). The other parameters are $\omega=0.05, \gamma_{1} / 2=-0.5, \gamma_{2} / 2=-0.5999$. The dashed lines in $(\mathrm{c}, \mathrm{d})$ show $|\cos (\Omega)|$ for the two orthogonal states of the Hermitian operator $H^{B}$.

on the environment into the system with the aim to accumulate as much as possible of the total coupling strength between system and environment onto one of the states (in the onechannel case). By this, this state becomes short-lived while the other one decouples more or less from the environment and becomes long-lived. These two states are not analytically connected to the original individual states of the system.

While the mathematical properties of the eigenvalues of $\mathcal{H}^{(2)}$ are studied in many papers for isolated EPs, their influence onto the vicinity of the EPs and onto the eigenfunctions is considered in only a few papers, see e.g. the review [10]. The interesting question how the ranges of different EPs may influence each other is not at all considered in the literature. It will be discussed in detail in the following paper [44] by using the results shown in Figs. 1] to 4 

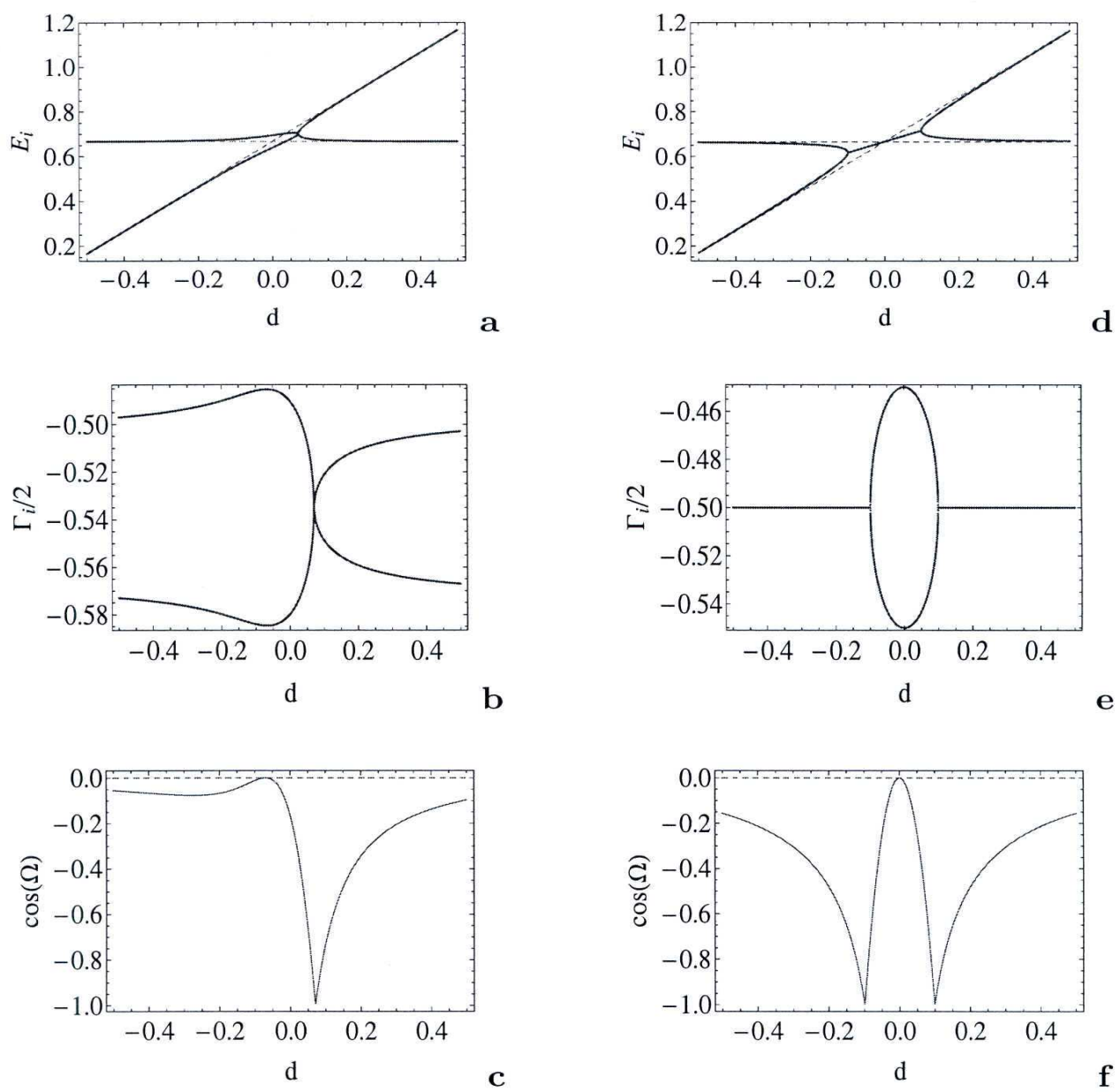

FIG. 4: The same as Fig. 3 a-c, but $\omega=0.05(1+i) / \sqrt{2}, \gamma_{1} / 2=-0.5, \gamma_{2} / 2=-0.57$ (left panel, a-c) and $\omega=0.05 i, \gamma_{1} / 2=-0.5, \gamma_{2} / 2=-0.5$ (right panel, d-f).

\section{CROSSING OF TWO STATES IN QUANTUM SYSTEMS WITH LOSS AND GAIN}

\section{A. Basic equations, Hamiltonian with loss and gain}

As has been shown in [40 43], the quantum mechanical Schrödinger equation and the optical wave equation in symmetric optical lattices are formally equivalent. Complex symmetric structures can be realized by involving symmetric index guiding and an antisymmetric gain/loss profile.

The main difference of these optical systems to open quantum systems consists in the symmetry of gain and loss in the first case while the states of an open quantum system can 
only decay $\left(\operatorname{Im}\left(\varepsilon_{1,2}\right)<0\right.$ and $\operatorname{Im}\left(\mathcal{E}_{1,2}\right)<0$ for both states $)$. Thus, the modes involved in the non-Hermitian Hamiltonian in optics appear in complex conjugate pairs while this is not the case in an open quantum system. As a consequence, the Hamiltonian for the description of the structures in optical lattices may have real eigenvalues in a large parameter range [34], similar as in, e.g., the papers [28-31]].

The $2 \times 2$ non-Hermitian Hamiltonian may be written, in this case, as [29, 30, 33]

$$
\mathcal{H}_{P T}=\left(\begin{array}{cc}
e-i \frac{\gamma}{2} & w \\
w & e+i \frac{\gamma}{2}
\end{array}\right),
$$

where $e$ stands for the energy of the two modes, $\pm \gamma$ describes gain and loss, respectively, and the real coupling coefficient $w$ stands for the coupling of the two modes via the lattice. When optical lattices are studied with vanishing gain, the Hamiltonian reads

$$
\mathcal{H}_{P T}^{\prime}=\left(\begin{array}{cc}
e-i \frac{\gamma}{2} & w \\
w & e
\end{array}\right) .
$$

In realistic systems, $w$ in (25) and (26) is mostly real (or at least almost real).

\section{B. Eigenvalues of the Hamiltonian with loss and gain}

The eigenvalues of the Hamiltonian (25) differ from (15),

$$
\mathcal{E}_{ \pm}^{P T}=e \pm \frac{1}{2} \sqrt{4|w|^{2}-\gamma^{2}} \equiv e \pm Z_{P T}
$$

A similar expression is derived in [29, 30]. Since $e$ and $\gamma$ are real, the $\mathcal{E}_{ \pm}^{P T}$ are real when $4|w|^{2}>\gamma^{2}$. Under this condition, the two levels repel each other in energy what is characteristic of discrete interacting states. When the interaction $w$ is fixed, the level repulsion decreases with increasing $\gamma$. When $4|w|^{2}=\gamma^{2}$ the two states cross. Here, $\mathcal{E}_{ \pm}^{P T}=e$ and $\gamma= \pm \sqrt{4|w|^{2}}$. With further increasing $\gamma$ and $4|w|^{2}<\gamma^{2}$ ( $w$ fixed for illustration), width bifurcation (called PT-symmetry breaking) occurs and $\mathcal{E}_{ \pm}^{P T}=e \pm \frac{i}{2} \sqrt{\gamma^{2}-4|w|^{2}}$.

These relations are in accordance with (6) to (10) for open quantum systems. Since $|w|$ is real, two EPs exist according to

$$
4|w|^{2}=( \pm \gamma)^{2}
$$


Further

$$
\begin{aligned}
& \gamma^{2}<4|w|^{2} \rightarrow Z_{P T} \in \Re \\
& \gamma^{2}>4|w|^{2} \rightarrow Z_{P T} \in \Im
\end{aligned}
$$

independent of the parameter dependence of $\gamma$.

In the case of the Hamiltonian (26), the eigenvalues read

$$
\mathcal{E}_{ \pm}^{\prime P T}=e-i \frac{\gamma}{4} \pm \frac{1}{2} \sqrt{4|w|^{2}-\frac{\gamma^{2}}{4}} \equiv e-i \frac{\gamma}{4} \pm Z_{P T}^{\prime} .
$$

We have level repulsion as long as $4|w|^{2}>\frac{\gamma^{2}}{4}$. While level repulsion decreases with increasing $\gamma$, the loss increases with increasing $\gamma$. At the crossing point, $\mathcal{E}_{ \pm}^{\prime P T}=e-i \frac{\gamma}{4}$. With further increasing $\gamma$ and $4|w|^{2} \ll \frac{\gamma^{2}}{4}$

$$
\mathcal{E}_{ \pm}^{\prime P T} \rightarrow e-i \frac{\gamma}{4} \pm i \frac{\gamma}{4}=\left\{\begin{array}{l}
e \\
e-i \frac{\gamma}{2}
\end{array}\right.
$$

The two modes (32) behave differently. While loss in one of them is large, it is almost zero in the other one. Thus, only one of the modes effectively survives. Equation (32) corresponds to high transparency at large $\gamma$.

Further, two EPs exist according to

$$
4|w|^{2}=( \pm \gamma / 2)^{2}
$$

and

$$
\begin{aligned}
& \gamma^{2} / 4<4|w|^{2} \rightarrow Z_{P T}^{\prime} \in \Re \\
& \gamma^{2} / 4>4|w|^{2} \rightarrow Z_{P T}^{\prime} \in \Im .
\end{aligned}
$$

In analogy to (28) up to (30) these relations are independent of the parameter dependence of $\gamma$.

Thus, there exist similarities between the eigenvalues $\mathcal{E}_{i}$ of $\mathcal{H}^{(2)}$ of an open quantum system and the eigenvalues of the Hamiltonian of a system with gain and loss. Interesting is the comparison of the eigenvalues $\mathcal{E}_{i}$ of $\mathcal{H}^{(2)}$ obtained for imaginary non-diagonal matrix elements $\omega$, with the eigenvalues of (25) or (26) for real $w$. In both cases, there are two EPs. In the first case, the energies $E_{i}$ are constant and the widths $\Gamma_{i}$ bifurcate between the 
two EPs. This situation is characteristic of an open quantum system at high level density with complex (almost imaginary) $\omega$, see Eqs. (8) to (10). In the second case however the difference $\left|E_{1}-E_{2}\right|$ in the energies increases (level repulsion) while the widths $\Gamma_{i}$ of both states are equal in the parameter range between the two EPs, see (28) to (301) and (33) to (35), respectively. Between the two EPs, level repulsion causes the two levels to be distant from one to another and $w$ is expected to be (almost) real according to (2) and (3). Formally, the role of energy and width is exchanged in the two cases.

It should be underlined here that the non-Hermitian Hamiltonian describing an open quantum system may also have real eigenvalues if certain conditions are fulfilled. Such a case is studied already more than 80 years ago [56], later in atomic physics [20, 21, 57, 58] and also in other systems such as double quantum dots [10, 25]. The so-called bound states in the continuum are caused by width bifurcation and, consequently, the width of the longlived resonance state may approach zero. This mechanism is different from that considered here since it creates real eigenvalues of the non-Hermitian Hamiltonian only at a few special parameter values.

\section{Eigenfunctions of the Hamiltonian with loss and gain}

The eigenfunctions of the two $2 \times 2$ Hamiltonians (25) and (26) show the same characteristic features as those of the Hamiltonian (4). The eigenmodes can be normalized, generally, according to (15) where $\Phi_{i}^{P T}\left(\Phi_{i}^{\prime} P T\right)$ denotes the right eigenmode. Far from an $\mathrm{EP}$, the eigenfunctions $\Phi_{i}^{P T}\left(\Phi_{i}^{\prime} P T\right)$ are orthogonal to one another. The orthogonality is lost in approaching the crossing point of the eigenvalue trajectories. Here, the modes show some skewness according to (16). As in the case of open quantum systems, the phase rigidity $r_{i}$ can be defined according to (22). It varies between 1 and 0 and is a quantitative measure for the skewness of the modes. Thus, the phases of the eigenmodes of the non-Hermitian Hamiltonians (25) and (26) are not rigid, and spectroscopic redistribution processes may occur under the influence of the environment (lattice).

The eigenfunctions $\Phi_{i}^{P T}$ of $\mathcal{H}_{P T}$ (and $\Phi_{i}^{\prime P T}$ of $\mathcal{H}_{P T}^{\prime}$ ) can be represented in a set of basic wavefunctions in full analogy to the representation of the eigenfunctions $\Phi_{i}$ of $\mathcal{H}^{(2)}$ in (23). They contain valuable information on the mixing of the wavefunctions under the influence of the non-diagonal coupling matrix elements $w$ in (25) and (26) , respectively, as well as its 
relation to EPs.

\section{Numerical results for a quantum system with loss and gain}

In realistic systems, the non-diagonal matrix elements $w$ of the non-Hermitian Hamiltonians (25) and (26) are real (or almost real) as follows from the level repulsion occurring between the two EPs (see above, Sect. [IIB). Nevertheless, we did some calculations also for complex and imaginary $w$ (results are not shown).

According to (25) and (26), the energies $e_{i}$ and widths $\gamma_{i}$ of the two states are the same. We choose $e_{1}=e_{2} \equiv e$ independent of the parameter $a$ in the considered region and $\gamma_{i}$ (gain and loss) to be parameter dependent.

In Fig. 5, the eigenvalues $\mathcal{E}^{P T}$ and $\mathcal{E}^{P T}$ of (25), left panel, and (26), right panel, are shown. The corresponding eigenfunctions shown in the lower part of Fig. 5, As can be seen from the results, the level repulsion appearing between the two EPs is accompanied by a complete (1:1) mixing of the eigenfunctions. The mixing vanishes only far from the EP (Figs. 5. e and $\mathrm{f}$ ).

This result is in full analogy to the results shown in Figs. 1,e,f and 2.e for open quantum systems with imaginary $\omega$ where width bifurcation is accompanied by a complete mixing of the eigenfunctions between the two EPs; and the mixing vanishes only far from the EPs. Further numerical studies have shown that also the phases of the eigenfunctions always jump by $\pi / 4$ at the EPs (not shown in Fig. 5).

We state therefore the following. The results of Fig. 5 obtained from calculations for systems with gain and loss and with real $w$ are formally similar to those received for open quantum systems with imaginary coupling coefficients $\omega$ (lower row in Figs. 1] and 2). In the two cases, the role of energy and width is formally exchanged.

In order to receive a better understanding of the role of gain in Fig. 5, we performed another calculation with slightly different energies $e_{i}$ of the two states. The results shown in Fig. 6] are very similar to those in Fig. 5, The differences are of the same type as those

obtained in corresponding calculations for open quantum systems with $\omega=0.05 i$, see Fig. 1 (left panel) in [48] with $\gamma_{1}=\gamma_{2}$ and Fig. 2 (left panel) in [48] with $\gamma_{1} \approx \gamma_{2}$, respectively.

Finally, we perform calculations with the Hamiltonian (4) but different signs for the two 

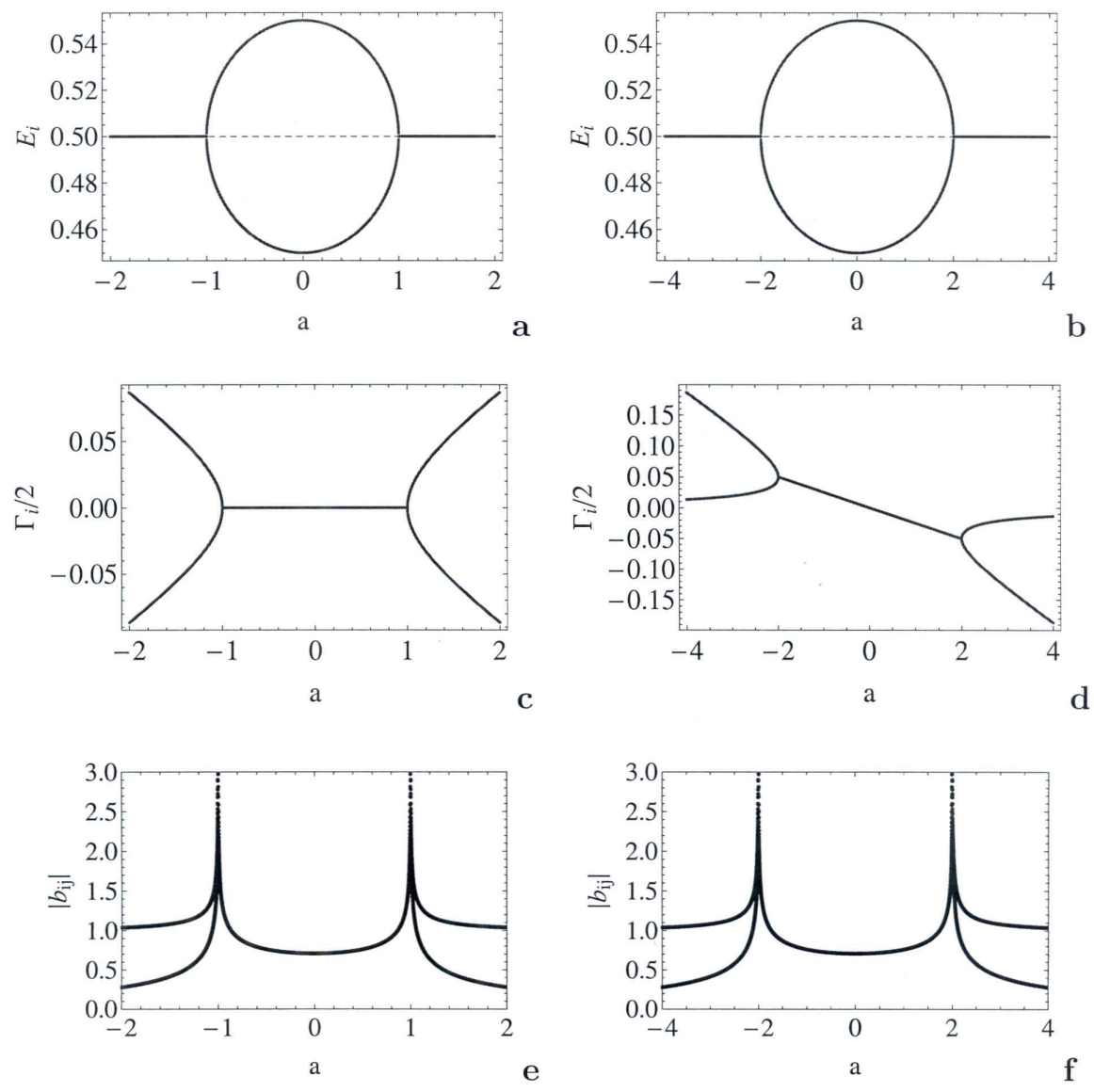

FIG. 5: Energies $E_{i}$ (top), widths $\Gamma_{i} / 2$ (mid) and mixing coefficients $\left|b_{i j}\right|$ (bottom) of the eigenfunctions $\Phi_{i}$ of $N=2$ states coupled to a common channel as a function of $a$. Parameters: $e=0.5 ; w=0.05$; $\gamma_{1} / 2=-0.05 a$; and $\gamma_{2}=-\gamma_{1}$ (left panel); $\gamma_{2}=0$ (right panel). In order to illustrate the symmetry properties, the results are shown for positive as well as for negative values $a$. The dashed lines in $(\mathrm{a}, \mathrm{b})$ show $e$.

$\gamma_{i}$ (and $\omega=\omega_{12}=\omega_{21}$ ). In this case, the eigenvalues $\mathcal{E}_{i, j} \equiv E_{i, j}+\frac{i}{2} \Gamma_{i, j}$ are given by (5) with

$$
Z=\frac{1}{2} \sqrt{\left(e_{1}-e_{2}\right)^{2}-\frac{1}{4}\left(\gamma_{1}-\gamma_{2}\right)^{2}+i\left(e_{1}-e_{2}\right)\left(\gamma_{1}-\gamma_{2}\right)+4 \omega^{2}} .
$$

According to the condition $Z=0$ for the appearance of an EP, we have one EP at the crossing point $a=a_{\mathrm{cr}}$ of the two $e_{i}$ trajectories (where $e_{1}(a)=e_{2}(a)$ ), if $\gamma_{1}=-\gamma_{2}$ is parameter independent and $\omega=\left|\gamma_{i} / 2\right|$ is real. There is however no EP when $\omega$ is imaginary. If $\omega$ is complex and the widths $\gamma_{i}$ of the two states have different signs, there is also one EP. We show the corresponding numerical results with one EP in Fig. 7.

We underline here that the results of Fig. 7 are obtained by using the Hamiltonian (44) for 

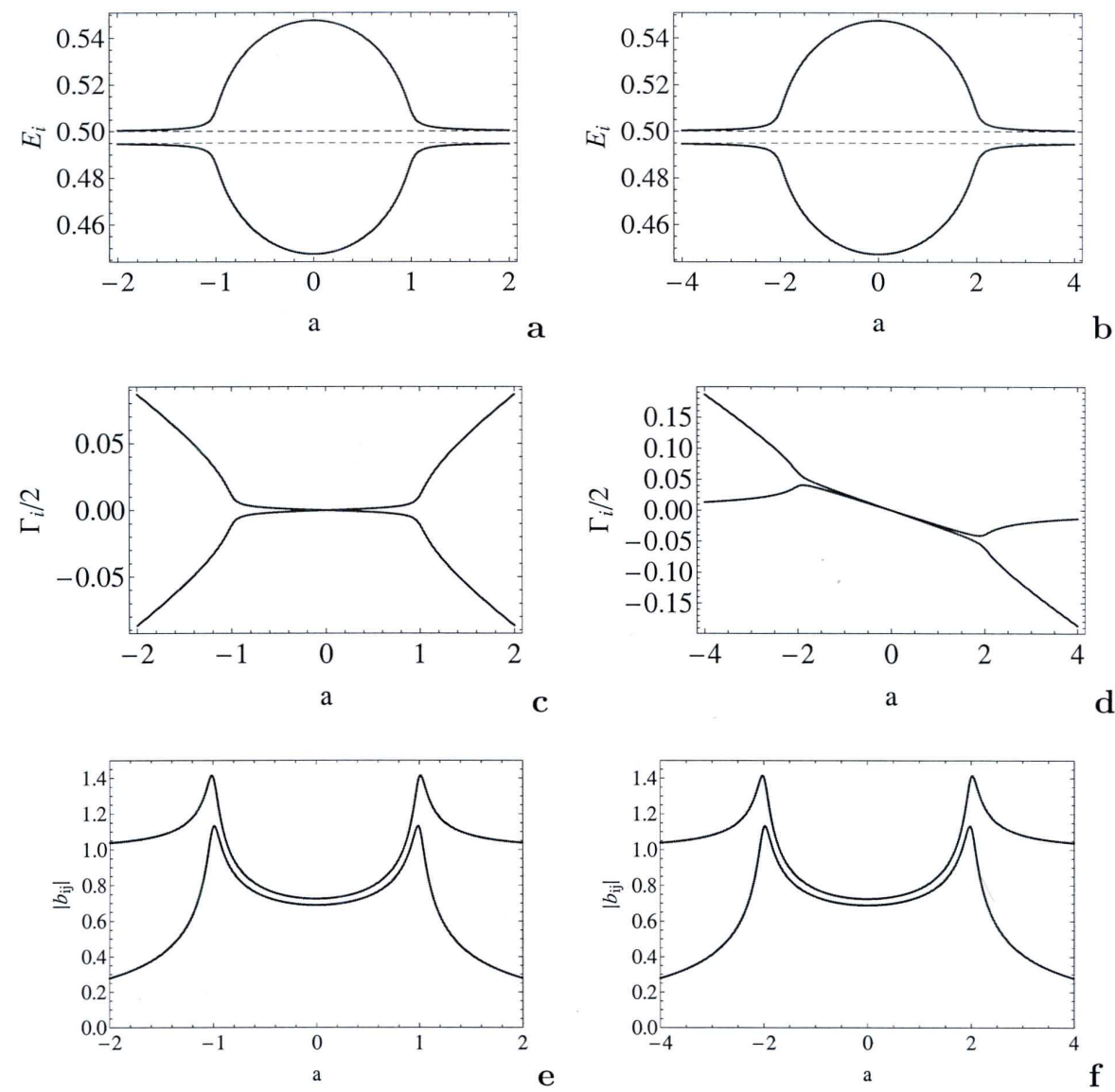

FIG. 6: The same as Fig. 5 but $e_{1}=0.500$ and $e_{2}=0.495$.

a system with parameter independent values of loss and gain. As usual, the EP appears at the crossing point of the energy trajectories if $\omega$ is real. The system shows the characteristic features of an open quantum system. A balance between gain and loss may appear, is however not necessary. Systems of this type will surely allow many different applications.

\section{CONCLUSIONS}

The results presented in the present paper show clearly the common features as well as the main difference between Hermitian and non-Hermitian quantum physics when describing small systems coupled to a small number of well-defined decay channels. Far from the singular EPs in non-Hermitian quantum physics, everything is analytical as in Hermitian quantum physics: Fermi's golden rule holds and counterintuitive results do not occur; the eigenfunctions of the Hamiltonian are nearly orthogonal; and the differences between 

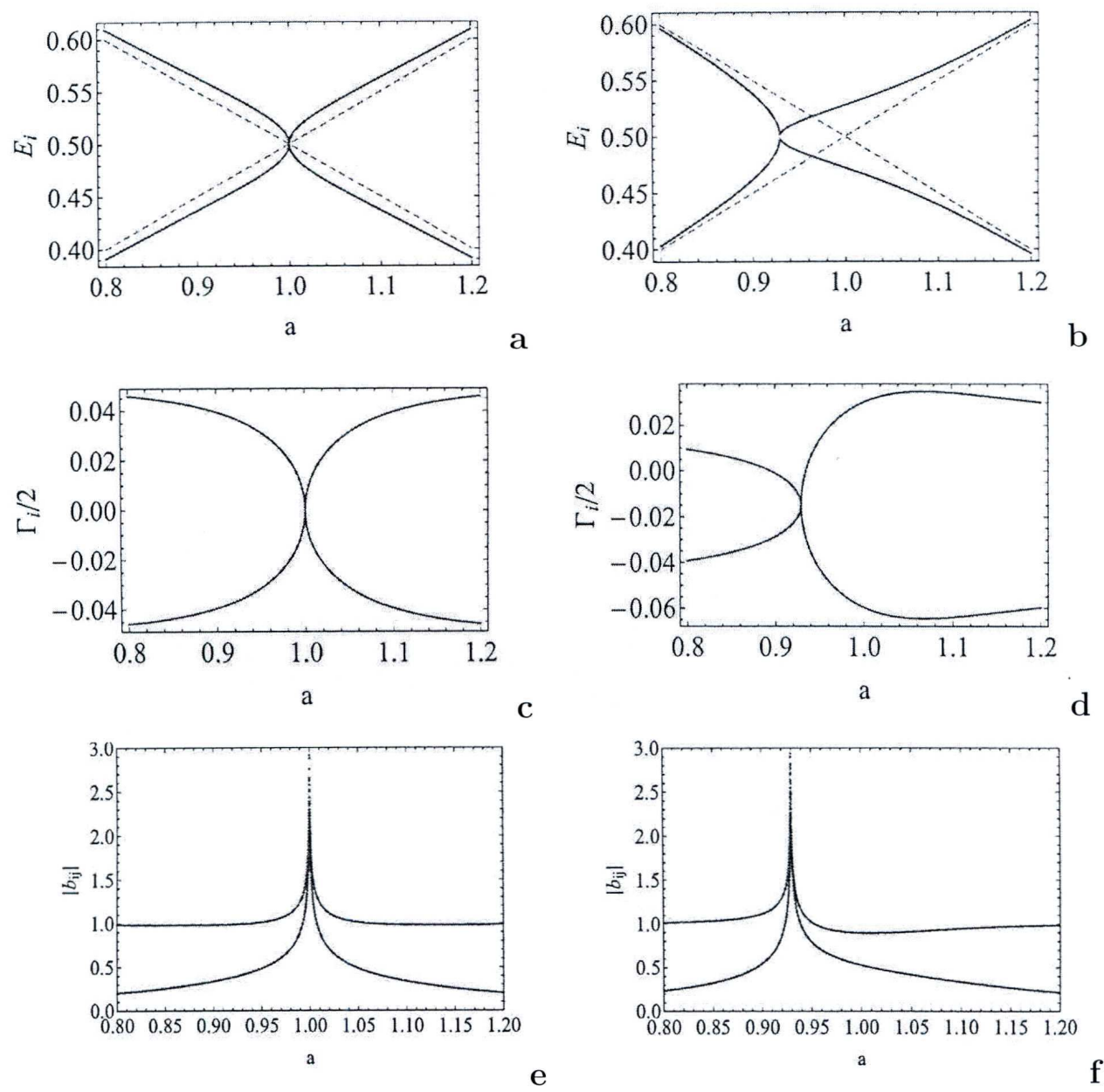

FIG. 7: Energies $E_{i}$ (top), widths $\Gamma_{i} / 2$ (mid) and mixing coefficients $\left|b_{i j}\right|$ (bottom) of $N=2$ states of an open quantum system with gain and loss which is coupled to one common channel, as a function of a. The parameters are $e_{1}=1-a / 2 ; e_{2}=a / 2$ and $\gamma_{1} / 2=-0.05 ; \gamma_{2} / 2=0.05 ; \omega=0.05$ (left panel); $\gamma_{1} / 2=-0.05 ; \gamma_{2} / 2=0.0205 ; \omega=0.05(1+i) / \sqrt{2}$ (right panel)

Hermitian and non-Hermitian quantum physics practically vanish. At (and near to) EPs, however, the functional change of the dependence of the observables changes radically. It is non-analytical and Fermi's golden rule does not hold. Instead, so-called counterintuitive results appear. This happens under the influence of the environment which is extremely large in the neighborhood of EPs where the eigenfunctions of the Hamiltonian are really biorthogonal. The environment itself represents an infinitely large number of degrees of freedom (continuum of scattering wavefunctions). It can be changed by means of external forces, however it can never be deleted from an open quantum system. Since all the individual states of the system are coupled to the common environment, their wavefunctions 
become mixed due to this coupling. Although this is a second-order process, it becomes the dominant one near to an EP.

This conclusion is based on the analytical and numerical results shown and discussed in the present paper. On the one hand, the differences between calculations with Hermitian and non-Hermitian Hamilton operator almost vanish far from EPs, see Fig. 3.,. On the other hand, counterintuitive results determine the dynamics of the system in the neighborhood of EPs. Most visible (and known for quite a long time) is the reduction of the lifetime of one of the two neighboring states in spite of increasing (imaginary) coupling strength between system and common environment. This result originates at the EP as all our calculations show.

The strong influence of an EP onto the dynamics of an open quantum system can be expressed quantitatively by the phase rigidity of the eigenfunctions of the non-Hermitian Hamilton operator which is defined in (22). The eigenfunctions are biorthogonal, and the phase rigidity vanishes in approaching an EP. Here, the wavefunctions differ from one another by only a phase factor. Such a result is, of course, completely different from that what is known in Hermitian quantum physics. It explains why the results obtained in non-Hermitian quantum physics differ substantially from those of Hermitian quantum physics only in the neighborhood of EPs.

The meaning of the environment for the physics of open quantum systems is confronted recently with existing experimental data in the review [59]. Further experimental and theoretical studies along the lines sketched in the present paper are necessary in order to receive more information on open quantum systems (which are embedded into a common well-defined environment) and to describe them by means of a non-Hermitian Hamilton operator. The results are basic also for a better understanding of processes occurring in optics, e.g. of the Dicke superradiance, as mentioned above. By choosing an appropriate environment, it is possible to manipulate the system and to produce, by doing this, systems with desired properties. These results are of importance for basic research as well as for applications. 
[1] M. Avinun-Kalish, M. Heiblum, O. Zarchin, D. Mahalu, and V. Umansky, Nature 436, 529 (2005)

[2] Focus on Interference in Mesoscopic Systems, New Journal of Physics 9, May 2007

[3] G. Hackenbroich, Phys. Rep. 343, 463 (2001)

[4] M. Müller and I. Rotter, Phys. Rev. A 80, 042705 (2009)

[5] G.A. Álvarez, E.P. Danieli, P.R. Levstein and H.M. Pastawski, J. Chem. Phys. 124, 194507 (2006)

[6] H.M. Pastawski, Physica B 398, 278 (2007)

[7] J. Lee, J.E. Han, S. Xiao, J. Song, J.L. Reno, and J.B. Bird, Nature Nanotechnology 9, 101 (2014)

[8] M. Cahay, Nature Nanotechnology 9, 97 (2014)

[9] I. Rotter, Rep. Prog. Phys. 54, 635 (1991)

[10] I. Rotter, J. Phys. A 42, 153001 (2009)

[11] N. Moiseyev, Non-Hermitian Quantum Mechanics, Cambridge University Press, 2011

[12] A.E. Miroshnichenko, S. Flach, Y.S. Kivshar, Rev. Mod. Phys. 82, 2257 (2010)

[13] Y. Yoon, M.G. Kang, T. Morimoto, M. Kida, N. Aoki, J.L. Reno, Y. Ochiai, L. Mourokh, J. Fransson, and J.P. Bird, Phys. Rev. X 2, 021003 (2012)

[14] C. Jung, M. Müller and I. Rotter, Phys. Rev. E 60, 114 (1999)

[15] A. Biella, F. Borgonovi, R. Kaiser, G.L. Celardo, Europhysics Letters (EPL) 103, 57009 (2013)

[16] R.H. Dicke, Phys. Rev. 93, 99 (1954)

[17] G.L. Celardo, F. Borgonovi, M. Merkli, V.I. Tsifrinovich, G.P. Berman, J. Phys. Chem. C 116, $22105(2012)$

[18] The coalescence of two eigenvalues of a non-Hermitian operator should not be confused with the degeneration of two eigenstates of a Hermitian operator. The eigenfunctions of two degenerate states are different and orthogonal while those of two coalescing states are biorthogonal and differ only by a phase, see Eqs. (15) to (18).

[19] T. Kato, Perturbation Theory for Linear Operators, Springer, Berlin 1966

[20] A.I. Magunov, I. Rotter, and S.I. Strakhova, J. Phys. B 32, 1669 (1999) 
[21] A.I. Magunov, I. Rotter, and S.I. Strakhova, J. Phys. B 34, 29 (2001)

[22] E.N. Bulgakov, I. Rotter, and A.F. Sadreev, Phys. Rev. E 74, 056204 (2006)

[23] E.N. Bulgakov, I. Rotter, and A.F. Sadreev, Phys. Rev. B 76, 214302 (2007)

[24] I. Rotter and A.F. Sadreev, Phys. Rev. E 71, 036227 (2005)

[25] I. Rotter and A.F. Sadreev, Phys. Rev. E 71, 046204 (2005)

[26] M.C. Toroker and U. Peskin, J. Phys. B 42, 044013 (2009)

[27] W.D. Heiss, M. Müller and I. Rotter, Phys. Rev. E 58, 2894 (1998)

[28] C.M. Bender, Rep. Progr. Phys. 70, 947 (2007)

[29] A. Guo, G.J. Salamo, D. Duchesne, R. Morandotti, M. Volatier-Ravat, V. Aimez, G.A. Siviloglou and D.N. Christodoulides, Phys. Rev. Lett. 103, 093902 (2009)

[30] C.E. Rüter, G. Makris, R. El-Ganainy, D.N. Christodoulides, M. Segev, and D. Kip, Nature Physics 6, 192 (2010)

[31] T. Kottos, Nature Physics 6, 166 (2010)

[32] J. Schindler, Z. Lin, J.M. Lee, H. Ramezani, F.M. Ellis, and T. Kottos, J. Phys. A 45, 444029 (2012)

[33] I. Rotter, J. Opt. 12, 065701 (2010)

[34] H. Eleuch and I. Rotter, Acta Polytechnica 54, 106 (2014); Int. J. Theor. Phys., DOI 10.1007/s10773-014-2375-3, (2015)

[35] B. Wahlstrand, I.I. Yakimenko, and K.F. Berggren, Phys. Rev. E 89, 062910 (2014)

[36] Y.N. Joglekar, C. Thompson, D.D. Scott, and G. Vemuri, Eur. Phys. J. Appl. Phys. 63, 30001 (2013)

[37] C.M. Bender, M. Gianfreda, S.K. Özdemir, B. Peng, and L. Yang, Phys. Rev. A 88, 062111 (2013)

[38] B. Peng, S.K. Özdemir, F.C. Lei, F. Monifi, M. Gianfreda, G.L. Long, S.H. Fan, F. Nori, C.M. Bender, and L. Yang, Nature Physics 10, 394 (2014)

[39] B. Peng, S.K. Özdemir, S. Rotter, H. Yilmaz, M. Liertzer, F. Monifi, C. M. Bender, F. Nori, and L. Yang, Science 346, 328 (2014)

[40] A. Ruschhaupt, F. Delgado and J.G. Muga, J. Phys. A 38, L171 (2005)

[41] R. El-Ganainy, K.G. Makris, D.N. Christodoulides and Z.H. Musslimani, Optics Lett. 32, $2632(2007)$

[42] K.G. Makris, R. El-Ganainy, D.N. Christodoulides and Z.H. Musslimani, Phys. Rev. Lett. 
100, $103904(2008)$

[43] Z.H. Musslimani, K.G. Makris, R. El-Ganainy and D.N. Christodoulides, Phys. Rev. Lett. 100, $030402(2008)$

[44] H. Eleuch and I. Rotter, Eur. Phys. J. D (2015)

[45] H. Feshbach, Ann. Phys. 5, 357 (1958) and 19, 287 (1962)

[46] H. Eleuch and I. Rotter, Phys. Rev. E 87, 052136 (2013)

[47] In difference to [46], the definitions $\epsilon_{i}=e_{i}+\frac{i}{2} \gamma_{i}$ and $\mathcal{E}_{i}=E_{i}+\frac{i}{2} \Gamma_{i}$ (with $\gamma_{i} \leq 0$ and $\Gamma_{i} \leq 0$ for decaying states) are used in the present paper

[48] H. Eleuch and I. Rotter, Eur. Phys. J. D 68, 74 (2014)

[49] L. Landau, Physics Soviet Union 2, 46 (1932)

[50] C. Zener, Proc. Royal Soc. London, Series A 137, 692 (1932)

[51] I. Rotter, Special Issue Quantum Physics with Non-Hermitian Operators: Theory and Experiment, Fortschr. Phys. 61, 178 (2013)

[52] I. Rotter, Phys. Rev. E 68, 016211 (2003)

[53] M. Müller, F.M. Dittes, W. Iskra, and I. Rotter, Phys. Rev. E 52, 5961 (1995)

[54] In studies of other researchers, the factor $i$ in (18) does not appear. This difference is discussed and compared with experimental data in the Appendix of [51] and in Sect. 2.5 of [10], see also Figs. 4 and 5 in [35].

[55] I. Rotter, Phys. Rev. E 64, 036213 (2001)

[56] J. von Neumann and E. Wigner, Phys. Zeitschr. 30, 465 (1929)

[57] H. Friedrich and D. Wintgen, Phys. Rev. A 31, 3964 (1985)

[58] H. Friedrich and D. Wintgen, Phys. Rev. A 32, 3231 (1985)

[59] I. Rotter and J.P. Bird, A Review of Progress in the Physics of Open Quantum Systems: Theory and Experiment, Rep. Prog. Phys. 78 (2015); Shortened version arXiv:1507.08478 (July 2015) 


\title{
Part II: Three and more states
}

\begin{abstract}
Using the formalism for the description of open quantum systems by means of a nonHermitian Hamilton operator, we study the occurrence of dynamical phase transitions as well as their relation to the singular exceptional points (EPs). First, we provide the results of an analytical study for the eigenvalues of three crossing states. These crossing points are of measure zero. Then we show numerical results for the influence of a nearby ("third") state onto an EP. Since the wavefunctions of the two crossing states are mixed in a finite parameter range around an EP, three states of a physical system will never cross in one point. Instead, the wavefunctions of all three states are mixed in a finite parameter range in which the ranges of the influence of different EPs overlap. We may relate these results to dynamical phase transitions observed recently in different experimental studies. The states on both sides of the phase transition are non-analytically connected.
\end{abstract}

\section{INTRODUCTION}

Phase transitions are studied since very many years in different physical systems. In spite of their special features characteristic of every system, common to all of them is that the states at one side of the transition are not analytically connected to those at the other side. Recently, phase transitions are observed experimentally and investigated theoretically also in small open quantum systems. Mostly, the results are counterintuitive, when considered from the point of view of Hermitian quantum physics. Some years ago, results of such a type could be explained qualitatively in the framework of non-Hermitian quantum physics by relating them to a phase transition occurring in an open quantum system [1]. At the same time, these results are related [2] to the existence of singular points [3], the so-called exceptional points (EP), emerging in the non-Hermitian formalism.

By now, we have a much better understanding of these phenomena, especially in the field of mesoscopic physics where it is possible to trace the phase transition by means of parameter variation. A detailed discussion can be found in the recent review [4] where theory based on non-Hermitian quantum physics, is confronted with known experimental results on open quantum systems. In recent papers, this type of phase transition is called mostly dynamical 
phase transition (DPT) in order to underline its relation to the dynamics of open quantum systems. Much less understood are similar phenomena in optics although they are known experimentally for a long time, e.g. the Dicke superradiance [5].

As a result of many differnt studies during last years (for references see [6]), the singular EPs of the non-Hermitian formalism play really an important role for the occurrence of phenomena called counterintuitive in the Hermitian formalism. In [6], the non-Hermitian formalism is sketched. It allows us to study not only the eigenvalues but also the eigenfunctions of a non-Hermitian Hamilton operator $\mathcal{H}$ in the neighborhood of the EPs.

At an EP, two eigenvalues of the Hamiltonian $\mathcal{H}$ coalesce while the corresponding eigenfunctions differ from one another only by a phase. Such a situation is possible only beyond standard Hermitian quantum physics since the eigenfunctions of all states of a Hermitian Hamilton operator are orthogonal (also when their eigenvalues accidentally coalesce), while those of a non-Hermitian operator are biorthogonal, and the phases of the eigenfunctions relative to one another may change.

In [6], analytical and numerical results for the eigenvalues as well as for the eigenfunctions of a non-Hermitian Hamiltonian are provided; the influence of EPs onto physical observables is discussed; the appearance of nonlinear terms in the Schrödinger equation around EPs is considered; as well as their relation to the phases of the two eigenfunctions relative to one another. The reduction of the phase rigidity of the eigenfunctions near to an EP means that the environment of scattering wavefunctions with its infinite large number of degrees of freedom plays here an extremely important role for the dynamics of the system since it mediates a coupling of the different states of the system via the environment. As a consequence, the wave functions of the two crossing states are mixed (via the environment) in a finite parameter range around an EP. They provide therefore valuable information on the environment not only at one point in the continuum (which is of measure zero), but in a finite parameter range around this point.

According to [6], the influence of EPs on the dynamical properties of open quantum systems consists in the following: (i) The eigenvalues of $\mathcal{H}$ show a non-analytical behavior (deviations from Fermi's golden rule) at and in the vicinity of an EP. Width bifurcation occurs due to $\operatorname{Im}(\omega)$, while level repulsion is caused by $\operatorname{Re}(\omega)$; (ii) The phases of the eigenfunctions are not rigid in a finite neighborhood of an EP, and the phase rigidity $r_{i} \rightarrow 0$ at the EP. This dynamical feature allows the environment to influence the system extremely 
strong at and near to an EP. These results hold true for open many-body quantum systems the states of which decay into the environment of scattering states, as well for those systems which may absorb particles from the environment.

The main interest of our present study on non-Hermitian quantum systems is to find an answer to the question how different EPs influence one another and how they are related to a DPT occurring in a physical system. Most studies have to be performed numerically, what is an expression of the well-known fact that the states below and beyond a phase transition are non-analytically connected. We restrict ourselves, in our paper, to the coherent (collective) phenomena induced in an open quantum system by embedding it into a common environment. These phenomena are very robust. We will not consider decoherent phenomena in the present paper which arise by coupling the system to a large nonspecific environment, since this coupling does not cause any global new features of the system such as e.g. a DPT, in which we are interested.

In our paper we consider first the crossing of the eigenvalues of three states analytically and discuss shortly their relevance for physical processes (Sect. II). In the following Sect. III] we investigate numerically the influence of a nearby state onto an EP. Here, we consider not only the eigenvalues but also the eigenfunctions of the non-Hermitian Hamilton operator. The numerical studies are performed for open quantum systems with emission (loss) of particles into the environment; and also for systems which additionally may absorb particles from the environment (systems with loss and gain).

In Sect. IV of the present paper, we address the problem of the relation of EPs to DPTs appearing in systems with more than two states. We are able to justify the restriction of the parameter dependence of the Hamiltonian $\mathcal{H}$ to its non-Hermitian part what allows us to receive quickly information on the most important global spectroscopic redistribution processes occurring in open quantum systems, i.e. on the main features of the DPTs. We discuss also numerical results obtained for systems with gain and loss and their relation to those obtained for open quantum systems. Some conclusions on the meaning of EPs for the dynamics of open quantum systems are drawn in Sect. V. Here, we point also to the meaning of the results for phenomena observed in optics, e.g. for the Dicke superradiance. 


\section{CROSSING OF $N=3$ STATES IN AN OPEN QUANTUM SYSTEM}

We consider a system consisting of three levels coupled to one common continuum of scattering wavefunctions. The Hamiltonian reads

$$
\mathcal{H}^{(3)}=\left[\begin{array}{ccc}
\epsilon_{1}=e_{1}+i \frac{\gamma_{1}}{2} & \omega_{12} & \omega_{13} \\
\omega_{21} & \epsilon_{2}=e_{2}+i \frac{\gamma_{2}}{2} & 0 \\
\omega_{31} & 0 & \epsilon_{3}=e_{3}+i \frac{\gamma_{3}}{2}
\end{array}\right]
$$

where $\omega_{23}=\omega_{32}=0$ is assumed by using the doorway picture [7]. For simplicity, we assume that all coupling coefficients are equal to one another, $\omega_{i j}=\omega$. They may be real or complex.

According to [8], the eigenvalues can be determined from the polynomial

$$
\lambda^{3}+R \lambda^{2}+S \lambda+T=0
$$

with

$$
\begin{aligned}
& R=-\left(\epsilon_{1}+\epsilon_{2}+\epsilon_{3}\right) \\
& S=\epsilon_{1} \epsilon_{2}+\epsilon_{1} \epsilon_{3}+\epsilon_{2} \epsilon_{3}-2 \omega^{2} \\
& T=\omega^{2} \epsilon_{2}+\omega^{2} \epsilon_{3}-\epsilon_{1} \epsilon_{2} \epsilon_{3}
\end{aligned}
$$

Eq. (2) can be transformed to

$$
y^{3}+p y+q=0
$$

with

$$
\begin{aligned}
& y=\lambda+\frac{R}{3} \\
& p=\frac{3 S-R^{2}}{3} \\
& q=\frac{2 R^{3}}{27}-\frac{R S}{3}+T
\end{aligned}
$$

Using (6), (17) and

$$
\begin{aligned}
& u=\sqrt[3]{-\frac{q}{2}+\sqrt{\left(\frac{p}{3}\right)^{3}+\left(\frac{q}{2}\right)^{2}}} \\
& v=-\frac{p}{3 u}
\end{aligned}
$$


the three eigenvalues read

$$
\begin{aligned}
& \lambda_{1}=\frac{u+v}{2}-\frac{R}{3} \\
& \lambda_{2}=-\frac{u+v}{2}-\frac{R}{3}+\frac{u-v}{2} i \sqrt{3} \\
& \lambda_{3}=-\frac{u+v}{2}-\frac{R}{3}-\frac{u-v}{2} i \sqrt{3}
\end{aligned}
$$

When $y=0$ and $q=0$ the three eigenvalues cross according to (5) and (44). Here

$$
\lambda_{1,2,3}=-\frac{R}{3}=\frac{\epsilon_{1}+\epsilon_{2}+\epsilon_{3}}{3}
$$

where the definition (3) of $R$ is used. Further,

$$
u=\sqrt{\frac{p}{3}} ; \quad v=-\sqrt{\frac{p}{3}}=-u .
$$

Equation (13) shows that the crossing point is determined by the distance $R$ between the three complex energies $\epsilon_{i}$ of the states. The way on which the crossing point is approached plays therefore an important role. When approaching the crossing point by means of $u=$ $-v \rightarrow 0$, the eigenvalues (10) to (12) at the crossing point read

$$
\begin{aligned}
& \lambda_{1}=-\frac{R}{3} \\
& \lambda_{2} \rightarrow-\frac{R}{3}+i u \sqrt{3} \\
& \lambda_{3} \rightarrow-\frac{R}{3}-i u \sqrt{3}
\end{aligned}
$$

The two eigenvalues $\lambda_{2}$ and $\lambda_{3}$ form an EP when $u \rightarrow 0$. The eigenvalue $\lambda_{1}$ is not influenced by this condition. It plays the role of an observer state that can be exchanged at the EP with one of the other two states, but does not participate in the spectroscopic redistribution processes taking place at the crossing point. When however $u=0$ and the crossing point is approached by varying another parameter (being independent of $u$ ), all three states participate in the spectroscopic redistribution processes.

The conclusion from this analytical study is the following. According to the parameter varied in approaching the crossing point of three states, two different types of crossing points exist. The differences between these two types of crossing points consist in the following

- when the crossing point is approached by $u \rightarrow 0$, two states show the signatures of an EP while the third state is an observer state that, generally, may exchange with one of the other states; 
- when the crossing point is approached by keeping constant $u=0$ and varying another independent parameter, the three states form together a common crossing point, at which all three states participate in the spectroscopic redistribution processes.

In any case, the spectroscopic redistribution caused by the crossing of three states is a nonlinear process according to (2). The results hold for systems with loss (all $\gamma_{i}$ negative) as well as for systems with loss and gain (negative and positive $\gamma_{i}$ ).

The detailed study of the crossing point of all three eigenvalues of the Hamiltonian (1) is of formal-mathematical interest because it is a point in the continuum and therefore of measure zero. The geometric phase related to the crossing point of three states is, in any case, different from that of an EP for the crossing of two levels. This holds true even in the case when the third state is an observer state since this state may exchange with each of the two other states. The geometric phase depends therefore on the number $N$ of crossing states - a result that is difficult to understand for a realistic physical system.

According to the results obtained and discussed in [6], any "third" state of a realistic physical system will cross or avoid crossing with one of the two states in the neighborhood of the EP whose wavefunction differs however from that of the original state (because the wavefunctions of both states are mixed with one another near to the EP). At each of the crossings, the corresponding geometric phase is well defined: it is the geometric phase of an EP related to two states, and differs therefore from the Berry phase of a diabolic point by a factor 2 (see $[9]$ ).

While the properties of isolated EPs are studied experimentally as well as theoretically in many papers, the influence of a nearby state onto an EP is not at all investigated, up to now. In this case, the areas of influence of different EPs are expected to overlap, and nonlinear terms will appear not only at the EPs themselves but, above all, in a finite vicinity of them. Analytical studies are restricted due to these nonlinearities. When combined with numerical studies they are expected to give reliable results, see the next section. 

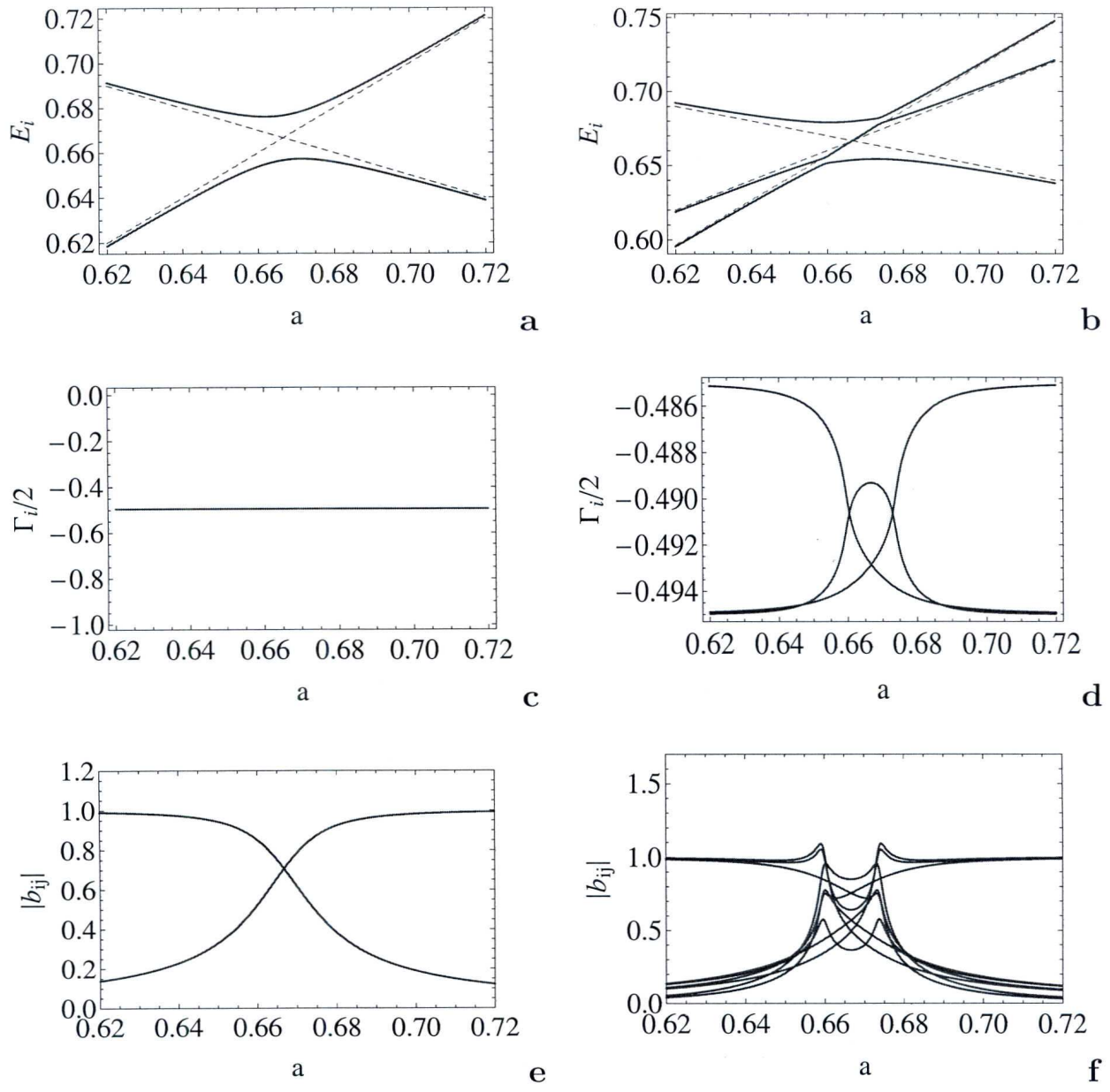

FIG. 1: Energies $E_{i}$ (top), widths $\Gamma_{i} / 2$ (mid) and mixing coefficients $\left|b_{i j}\right|$ (bottom) of the eigenfunctions $\Phi_{i}$ of $N=2$ (left panel a,c,e) and $N=3$ (right panel b,d,f) states of an open system coupled to a common channel by $\omega=0.01$ as a function of $a$. Parameters: $e_{1}=1-1 / 2 a ; \quad e_{2}=a ; \quad e_{3}=-1 / 3+3 / 2 a(\mathrm{~b}, \mathrm{~d}, \mathrm{f})$; $\gamma_{1} / 2=\gamma_{2} / 2=-0.495 ; \quad \gamma_{3} / 2=-0.485(\mathrm{~b}, \mathrm{~d}, \mathrm{f})$. The dashed lines in $(\mathrm{a}, \mathrm{b})$ show $e_{i}(a)$.

\section{INFLUENCE OF A NEARBY STATE ONTO AN EXCEPTIONAL POINT}

\section{A. Open quantum system with three nearby states}

The results of numerical calculations shown in Figs. 1 to 4 are performed with the nonHermitian Hamiltonian (11) for an open quantum system and with Eq. (24) of [6] for the coupling coefficients $\omega$. We assume that only the energies $e_{i}$ of the states depend on a parameter $a$ while the widths $\gamma_{i}$ are constant in the considered parameter range. We show 

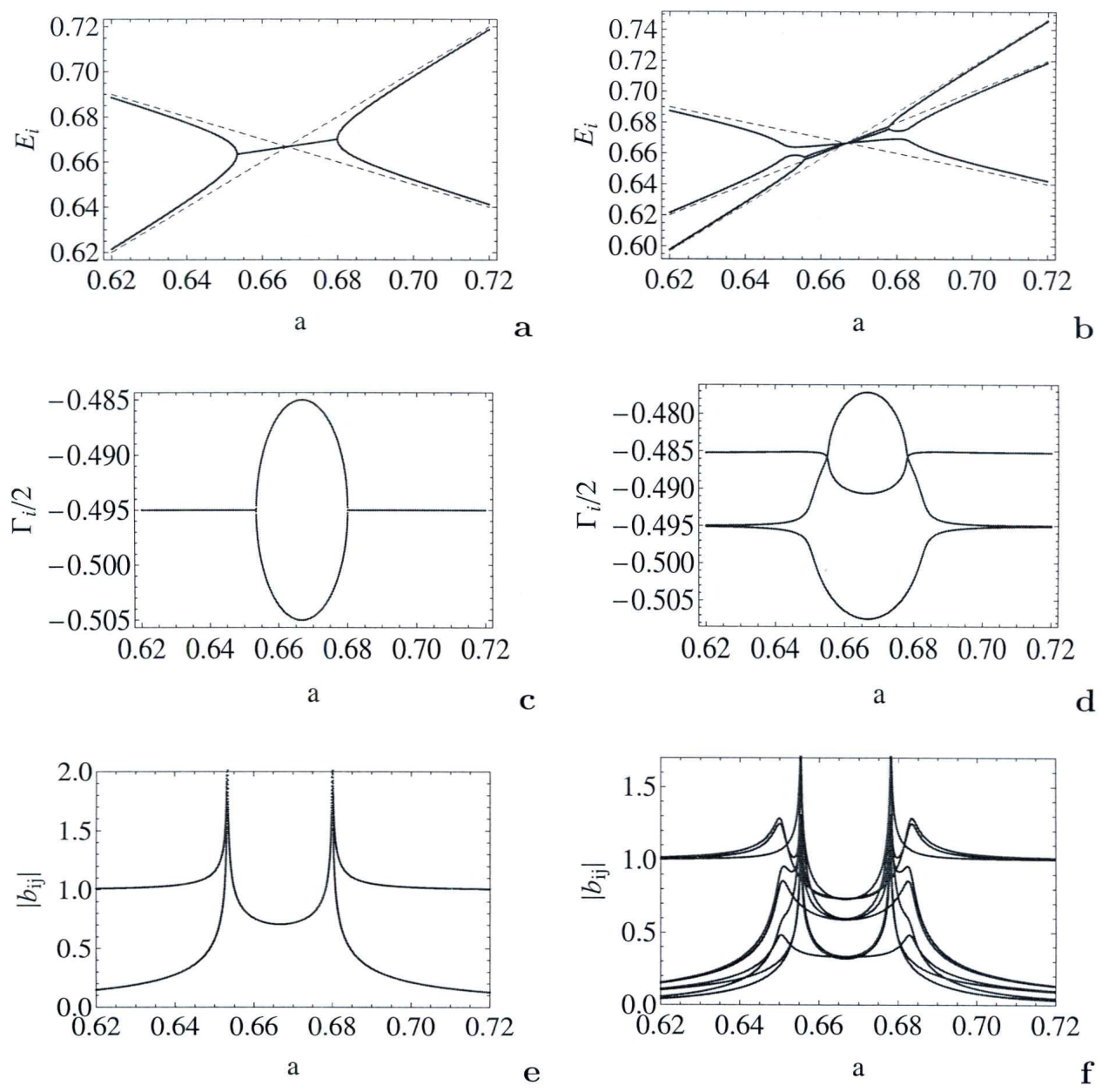

FIG. 2: The same as Fig. 1 but $\omega=0.01 i$ and $\gamma_{3} / 2=-0.4853(\mathrm{~b}, \mathrm{~d}, \mathrm{f})$.

the eigenvalues $\mathcal{E}_{i}=E_{i}+i / 2 \Gamma_{i}$ and the mixing coefficients $\left|b_{i j}\right|$ of the eigenfunctions $\Phi_{i}$ of (1) for real coupling coefficient $\omega$ (Fig. 1) as well as for imaginary $\omega$ (Fig. 21) and compare them with those of $\mathcal{H}^{(2)}$, Eq. (4) of [6], in the neighborhood of the EP.

In the left panel of Fig. 1 (real $\omega$ ), we see the typical avoided crossing of two levels with an exchange of the wavefunctions around the critical parameter value $a=a_{\mathrm{cr}}=2 / 3$ (see Sect. IID of [6] ). The right panel of Fig. 1 shows the crossing of the two levels with a third one. Here, two intersections can be seen: the first one at $a=a_{1}=0.65775<a_{\text {cr }}$ and the second one at $a=a_{2}=0.675>a_{\mathrm{cr}}$. At the two intersections $\left|b_{i j}\right|>1$ what is a clear hint to the existence of an EP. The eigenfunctions are mixed not only in the parameter range between the two intersections but also in a comparable large parameter range beyond them. As can be seen from the eigenvalue pictures Fig. 1.b,d the third state interacts with the two 

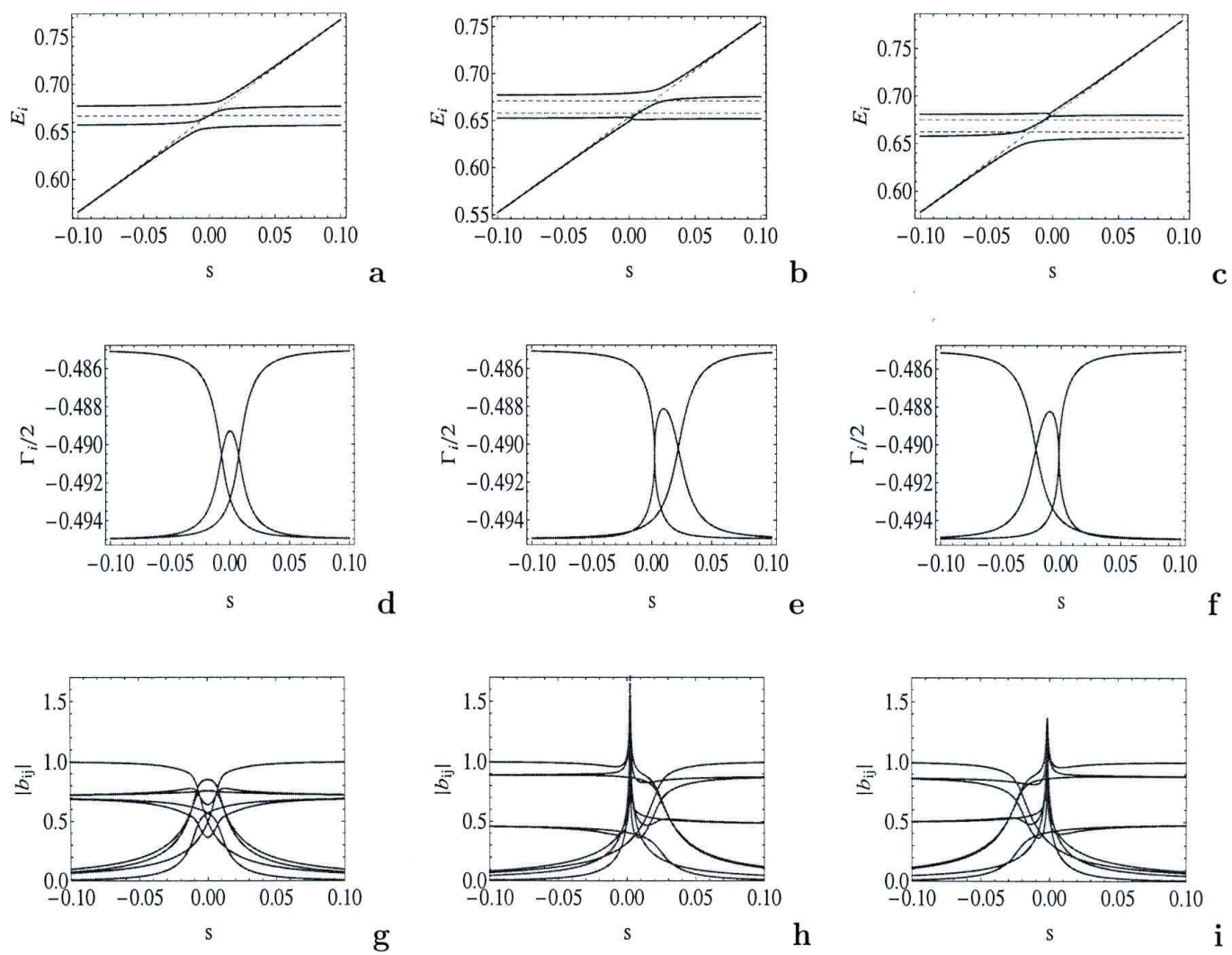

FIG. 3: Energies $E_{i}$ (top), widths $\Gamma_{i} / 2$ (mid) and mixing coefficients $\left|b_{i j}\right|$ (bottom) of $N=3$ states of an open system coupled to one common channel as a function of $s$ with $e_{3}=s-1 / 3+3 / 2 a$. $\omega=0.01$; $a=a_{c r}=2 / 3$ (left); $a=a_{1}=0.6539<a_{c r}$ (mid); $a=a_{2}=0.675>a_{c r}$ (right). The $e_{1}, e_{2}, \gamma_{1}, \gamma_{2}, \gamma_{3}$ are the same as in Fig. 1, The dashed lines in $(\mathrm{a}, \mathrm{b}, \mathrm{c})$ show $e_{i}(s)$.

other ones and exchanges with them.

Fig. 2 shows the results obtained with imaginary $\omega$. In the left panel, we see two EPs at $a=a_{1}=0.6539<a_{\mathrm{cr}}$ and $a=a_{2}=0.6774>a_{\mathrm{cr}}$, respectively. The appearance of two EPs and the width bifurcation between them is characteristic for two levels having the same (or similar) widths $\gamma_{i}$ (see Sect. IID of [6]). In the right panel of Fig. 2, a third level crosses the energy of the two states in the parameter range in which the widths of the two states bifurcate. Width bifurcation occurs now with participation of all three states (Fig. 2]d). Altogether, the width bifurcation is stronger than in the two-level case. At $a=a_{\mathrm{cr}}$, the difference between the largest and smallest values of $\Gamma_{i} / 2$ is 0.02 in the two-level case and 0.03 in the three-level case. Here, the widths of the three levels appear in two groups: 

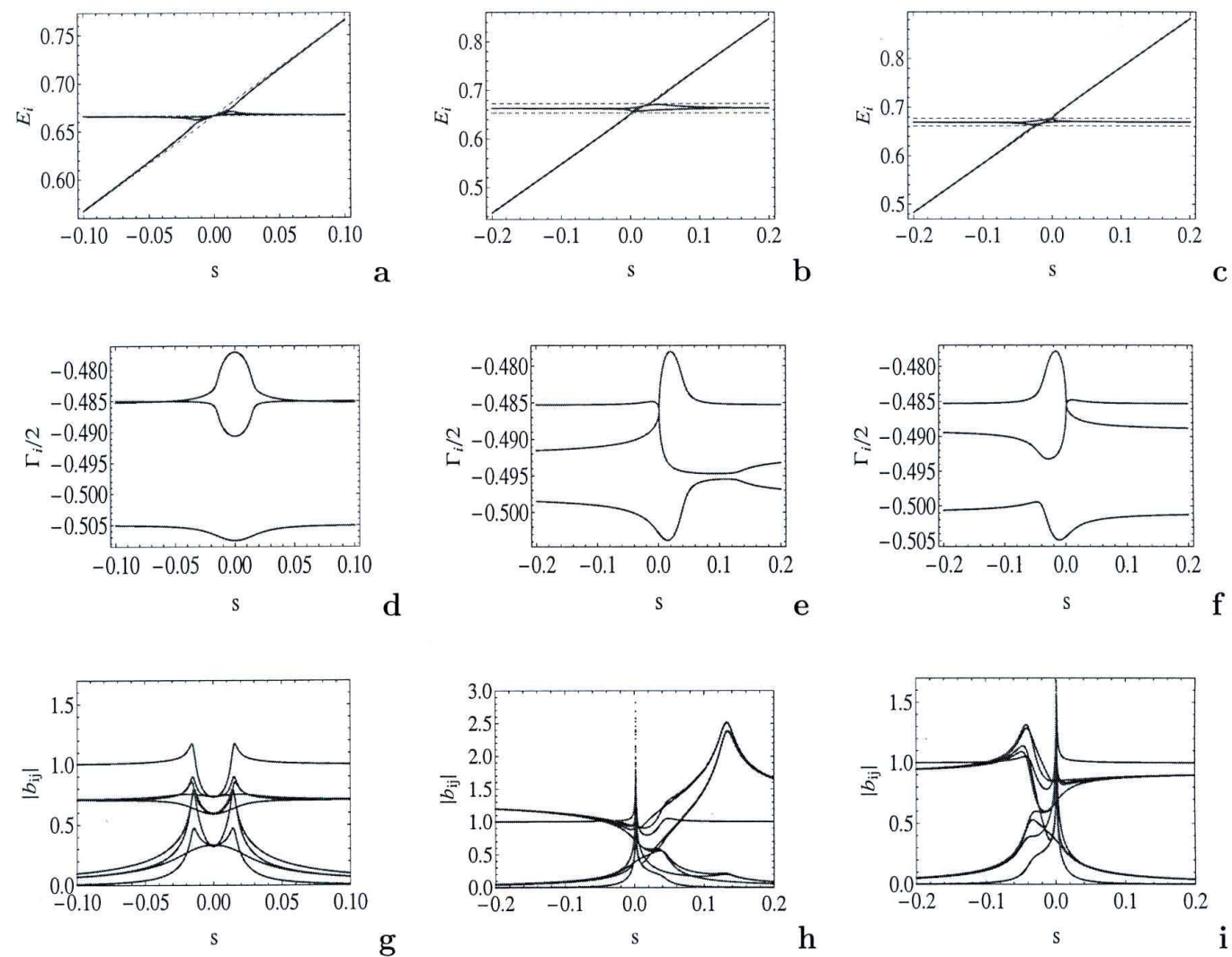

FIG. 4: Energies $E_{i}$ (top), widths $\Gamma_{i} / 2$ (mid) and mixing coefficients $\left|b_{i j}\right|$ (bottom) of $N=3$ states of an open system coupled to one common channel as a function of $s$ with $e_{3}=s-1 / 3+3 / 2 a$. $\omega=0.01 i$; $a=a_{c r}=2 / 3$ (left); $a=a_{1}=0.6539<a_{c r}$ (mid); $a=a_{2}=0.6774>a_{c r}$ (right). The $e_{1}, e_{2}, \gamma_{1}, \gamma_{2}, \gamma_{3}$ are the same as in Fig. 2, The dashed lines in $(\mathrm{a}, \mathrm{b}, \mathrm{c})$ show $e_{i}(s)$.

the largest width is much larger than the two other widths which, on their part, differ by a comparable small value from one another. This result is in agreement with the analytical result that the widths bifurcate at every eigenvalue crossing.

As in the case of real coupling coefficients $\omega$ (Fig. 1), the eigenfunctions obtained with imaginary $\omega$ (Fig. 2) are mixed strongly in the parameter range between the two EPs as well as beyond this range. The mixing is symmetrical around the critical parameter value $a=a_{\mathrm{cr}}$ in both cases and the large $\left|b_{i j}\right|$ point clearly to the existence of EPs.

In order to see more clearly the influence of a third level onto an avoided level crossing or an EP, we keep fixed all the parameters used in Figs. 1 and 2 with the exception of the energy $e_{3}$ of the third state. We choose $e_{3}=s-1 / 3+3 / 2 a$ and trace the eigenvalues and 
eigenfunctions of (11) as a function of $s$. At the value $a=a_{\mathrm{cr}}=2 / 3$, the three levels with energies $e_{i}$ cross (see Figs. 1., b and 2, b).

We show the results for different $a$ in Figs. 3 (real $\omega$ ) and 4 (imaginary $\omega$ ). The energies $E_{i}$ show level repulsion when $\omega$ is real, while the widths $\Gamma_{i}$ of all three states bifurcate when $\omega$ is imaginary. The eigenfunctions $\Phi_{i}$ are mixed strongly nearby the intersection points and the EPs, respectively. Some mixing remains at large values of $|s|$. The eigenvalue and eigenfunction pictures are completely symmetric around $s=0$ when $a=a_{c r}$ while this is not the case when $a \neq a_{\mathrm{cr}}$, neither for real nor for imaginary $\omega$. These last cases are, of course, more realistic than the symmetric one and will appear in physical systems. Comparing the results for different $a$, one sees the sensitive parameter dependence of the results in the critical region.

All the results shown in Figs. 1 to 4, are obtained for the case that the widths of two states are equal, $\gamma_{1}=\gamma_{2}$. Very similar results are obtained when $\gamma_{1} \approx \gamma_{2}$. Further, the results of calculations with complex $\omega$ (not shown) show characteristic features of the calculations with real as well as of those with imaginary $\omega$ (similar as in Figs. 1 and 2 in [6] ).

\section{B. Three nearby states in a system with loss and gain}

In Figs. 5 and 6 we show the influence of a nearby state onto an EP when the system has gain as well as loss and the coupling coefficient $w$ is real. Instead of width bifurcation occurring in the case of an open quantum system and imaginary coupling coefficient $\omega$, we see now the separation of the states from one another in energy. However, the third state has a large influence on the eigenvalue trajectories as well as on the parameter range in which the eigenfunctions of $\mathcal{H}$ are mixed.

In Fig. 5 the widths $\gamma_{i}$ of the states 2 and 3 increase with $a$, while that of state 1 decreases

with $a$. Due to this, there are altogether four EPs, at $a \approx-4 ;-2 ;+2 ;+4$. At $a \approx-4$, the widths $\Gamma_{i}$ of the two states with $\gamma_{i} \propto a$ separate from one another while their energies $E_{i}$ coalesce. The opposite happens at $a \approx-2$ : the width $\Gamma_{i}$ of the state with $\gamma_{i} \propto-a$ coalesces with that of one of the two states with $\gamma_{i} \propto a$ while the two states separate in energy at these critical $a$ value. The figure is symmetrical around $a=0$. The eigenfunctions of $\mathcal{H}$ are mixed in a parameter range, which is much larger than the range of mixing without the third state. 

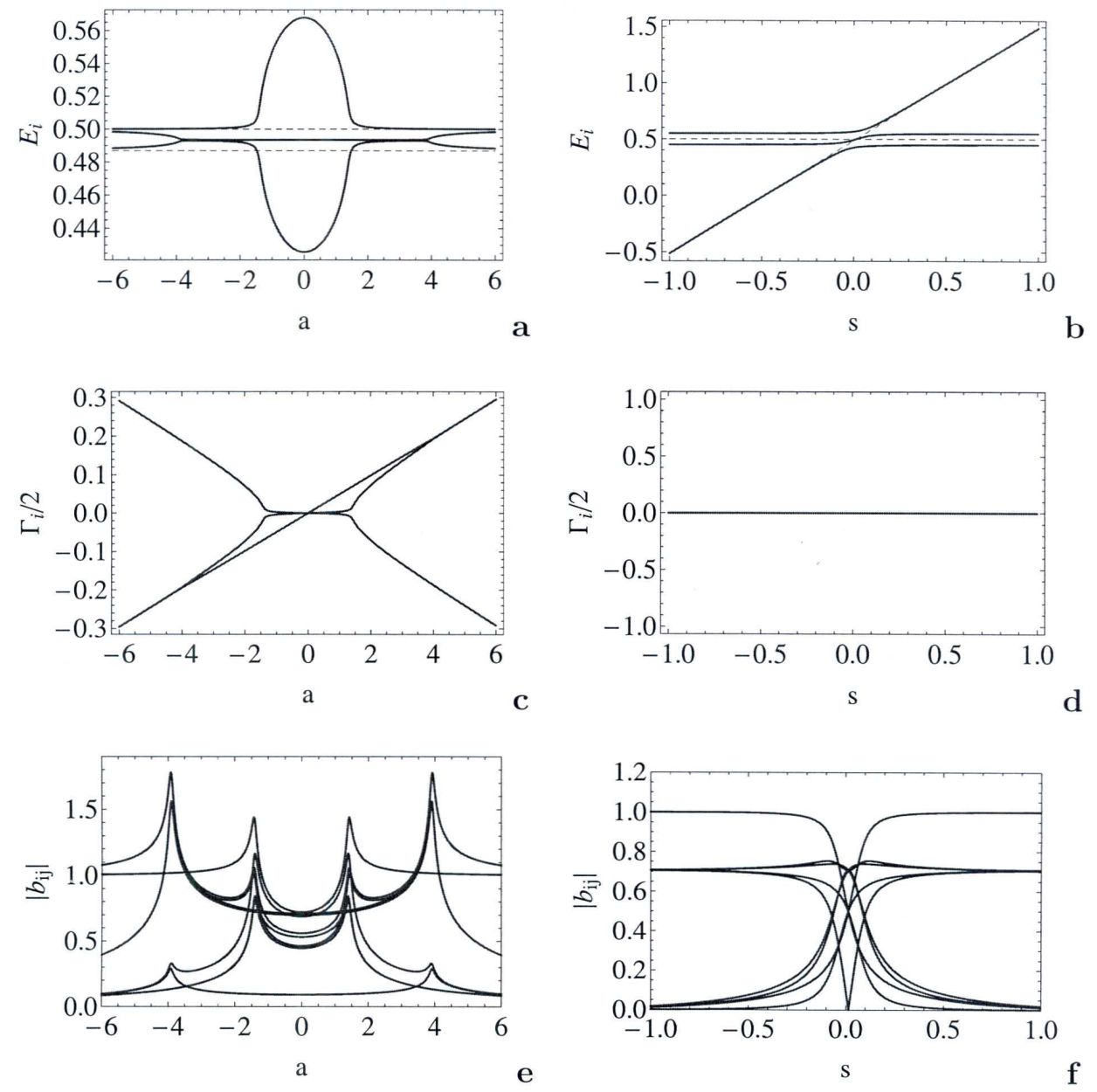

FIG. 5: Energies $E_{i}$ (top), widths $\Gamma_{i} / 2$ (mid) and mixing coefficients $\left|b_{i j}\right|$ (bottom) of $N=3$ states of a system with loss and gain, which is coupled to one common channel, as a function of $a$ (left panel) and $s$ (right panel). The parameters are $e_{1}=e_{2}=0.5$ and $e_{3}=0.487$ (left panel), $e_{3}=0.487+s ; a=0$ (right panel); $\gamma_{1} / 2=-0.05 a ; \gamma_{2} / 2=0.05 a ; \gamma_{3} / 2=0.05 a ; \quad w=0.05$. The dashed lines in $(\mathrm{a}, \mathrm{b})$ show $e_{i}(a)$.

In the right panel of Fig. 5, the influence of the third state onto the eigenvalues and eigenfunctions of $\mathcal{H}$ is shown by varying its distance $s$ to the two crossing states. The calculation is performed with $a=0$ and the results are symmetrical around $s=0$.

In Fig. 6, we show results for the case that the width of the third state is independent of the parameter $a$. It is $\gamma_{1} / 2=-\gamma_{2} / 2=-0.05 a, \gamma_{3} / 2=0.05$ (left panel) and $\gamma_{1} / 2=$ $-0.05 a ; \gamma_{2} / 2=\gamma_{3} / 2=0$ (right panel). The figures are similar to one another. In both cases, we have two EPs. The wavefunctions are strongly mixed between the two EPs as well as in a finite parameter range beyond them. 

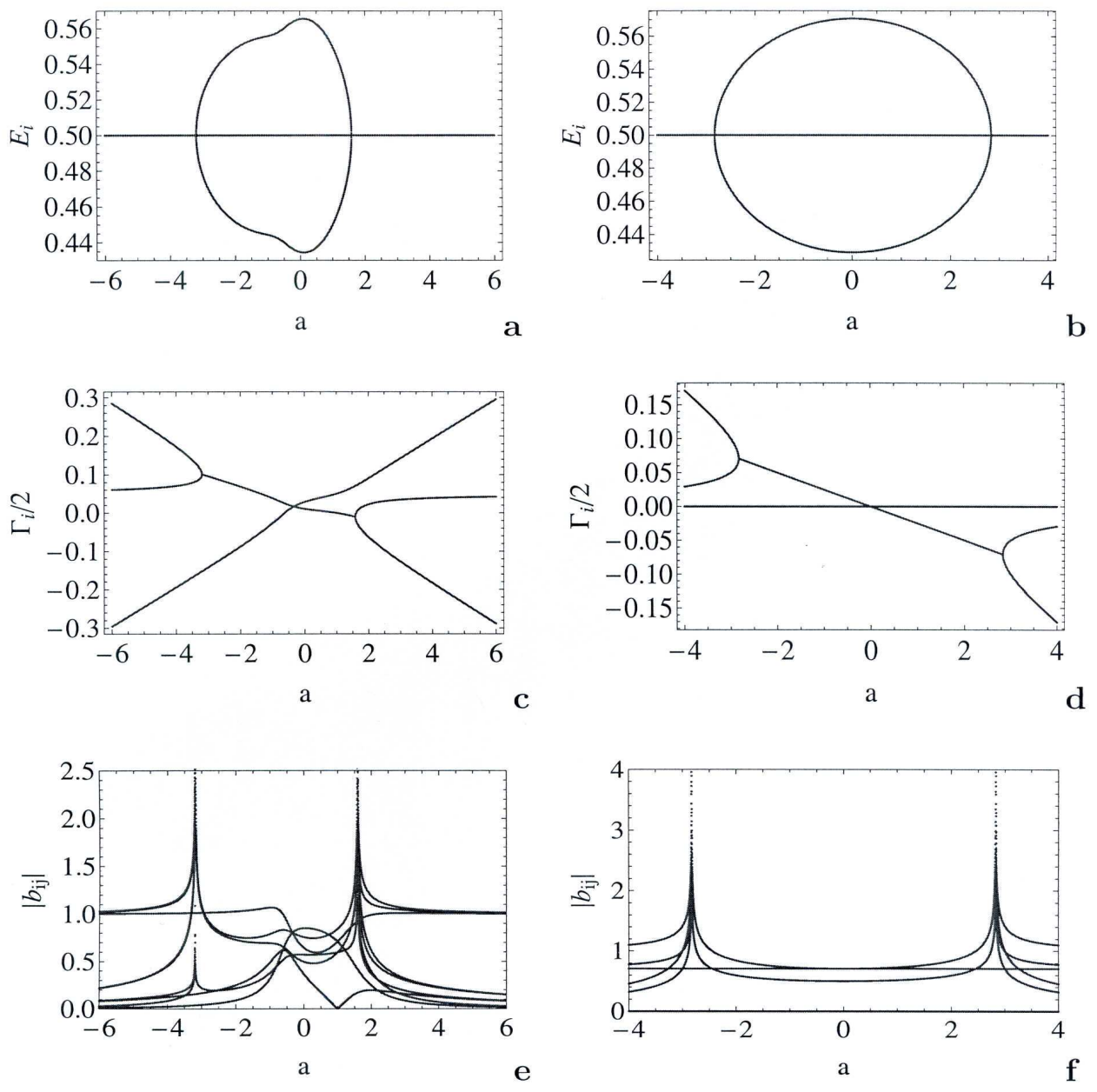

FIG. 6: Energies $E_{i}$ (top), widths $\Gamma_{i} / 2$ (mid) and mixing coefficients $\left|b_{i j}\right|$ (bottom) of $N=3$ states of a system with loss and gain, which is coupled to one common channel, as a function of $a$. The parameters are $e_{1}=e_{2}=e_{3}=0.5$ and $\gamma_{1} / 2=-0.05 a ; \gamma_{2} / 2=0.05 a ; \gamma_{3} / 2=0.05$ (left panel); $\gamma_{1} / 2=-0.05 a ; \quad \gamma_{2} / 2=$ $\gamma_{3} / 2=0$ (right panel); $\quad w=0.05$.

\section{CROSSING OF THREE (AND MORE) STATES AND DYNAMICAL PHASE TRANSITIONS}

In the foregoing sections of the present paper, we showed the influence of a nearby state onto an EP. First we considered the problem analytically for three different eigenvalues without paying attention to the corresponding eigenfunctions (Sect. III). As a result, the eigenvalues of all three states may coalesce in one point either by forming an EP (with all its signatures) of two of the states while the remaining state is uninvolved; or by forming a 
new common singular point. In both cases, the geometric phase differs from that of an EP since the states can be exchanged, even the "uninvolved" state may exchange with one of the other two states. A similar result for the geometric phase has been obtained in [10]. We found further that it depends on the manner the crossing point is approached which of the two possibilities will be realized.

However, these results cannot give any answer to the question how different EPs influence one another. More important than the crossing points themselves is their influence onto the system properties in some finite parameter range around an EP. All our calculations (Figs. 1] to 6) have shown the generic feature of three nearby states: the areas of influence of different EPs overlap and amplify, collectively, their impact onto physical values. The eigenfunctions $\Phi_{i}$ are mixed in the basic wavefunctions $\Phi_{j}^{0}$ (see Eq. (23) in [6]) in a finite critical parameter range. The range is larger than in the 2-level case. Also the width bifurcation (when the $\omega$ are imaginary and $\left.\gamma_{i}<0\right)$ is larger in the 3-level case than in the 2-level case (Fig. 2). It occurs stepwise and may explain, in this manner, the hierarchical trapping of resonance states found many years ago [11].

The conclusion from our numerical studies is that, in systems with three states, the various states lose their individual character in some critical parameter range, over which the distinct regions of influence of the various EPs overlap. The same holds true when the number of states is larger than three, as further numerical studies for four and more states have shown. The significance of the individual EPs is therefore lost in a finite parameter range, so that more than two states of a realistic physical system are unable to coalesce at a single point (remember that an EP is a point in the continuum and its influence on physical observables can be seen only in its vicinity). While in the case of an isolated EP the two states are exchanged at the EP (in accordance with the predictions of Eq. (18) in [6]), in the case with overlapping areas of influence of EPs, the states are no longer directly related to the original ones. Instead a spectroscopic redistribution, caused by several EPs, takes place that is aimed at achieving a dynamical stabilization of the system by accumulation as much coupling strength between system and environment as possible onto just one specific state (in the one-channel case) of the localized system. At the same time, the remaining states decouple strongly from the environment. The phase rigidity of the eigenfunctions is found to be reduced over a relatively large parameter range, where also significant nonlinearities appear. As mentioned already above, these effects persist when the number of interacting states is 
increased beyond three. It should be underlined here that this process of stabilization of the system as a whole occurs with the participation of all states and that, in the two-level case, it corresponds to nothing but width bifurcation.

In any case, the process of stabilization is directly related to the influence of the environment, with its infinitely large number of degrees of freedom, on the system at EPs. It may be related therefore to a DPT (see [1]). The relation between DPTs and EPs can be seen clearly only in the two-level case involving just a single EP. In the many-level case, the role of the EPs is somewhat hidden because the areas influenced by different EPs overlap and the positions of the EPs cannot be determined analytically. The numerical calculations show, however, clearly that they are responsible for the spectroscopic reordering processes which finally cause the DPT. This can be seen in Figs. 1 and 2 in which the eigenvalues and eigenfunctions of $\mathcal{H}$ are compared for the three-level case with those for the much simpler two-level case. According to these results, the non-Hermitian Hamilton operator of the open quantum system can be approximated, in the region of the DPT, quite well by

$$
\mathcal{H}=H^{B}-i \alpha V V^{+}
$$

where the non-Hermitian perturbation $V V^{+}$stands for the residuum, Eq. (3) of [6] , and the principal value integral, Eq. (2) of [6], is neglected (since its value is small when many states are nearby, what is the case in the range over which the DPT occurs). Many calculations are performed, indeed, by using the approximation (18) and tuning the parameter $\alpha$. The common feature of open quantum systems is that their quantum dynamics exhibits nonanalytically connected states on either side of the transition.

Summarizing, our results obtained for $N>2$ decaying states (with "loss") allows us to state that the basic features of a DPT can be seen already at a relatively small number of nearby states. A DPT is caused by several EPs (the crossing points of two resonance states) the areas of influence of which overlap. Fermi's golden rule holds only far from the DPT.

Similar results hold for systems in which the environment allows not only loss of particles but also gain (absorption of particles from the environment). The main difference to a natural system with only loss of particles (corresponding to an emission into the environment) is, obviously, that the coupling strength to the environment gives an additional real (Hermitian) part to the non-Hermitian Hamilton operator. This part causes level repulsion over a finite parameter range, and a balance between loss and gain becomes possible. 


\section{CONCLUSIONS}

Concluding we state the following. The present-day high resolution experimental studies require a description of quantum systems by taking into account their embedding into the common continuum of scattering wavefunctions. This natural environment exists always. It can be changed by means of external fields, however it can never be deleted. This basic assumption of the description of open quantum systems, used in the present paper, is proven experimentally, see for example [12] where it is shown that the interaction between two remote quantum states in semiconductor nanostructures (quantum point contacts) is essentially mediated by the continuum. The coupling of the open quantum system to the environment causes the Hamiltonian to be non-Hermitian whose eigenvalues $\mathcal{E}_{i}$ are complex, generally. They provide not only the energies $E_{i}=\operatorname{Re}\left(\mathcal{E}_{i}\right)$ of the states of the system but also their lifetimes which are inverse proportional to the widths $\Gamma_{i} / 2=\operatorname{Im}\left(\mathcal{E}_{i}\right)$. The feedback of the common environment onto the system is involved in the non-Hermitian Hamiltonian $\mathcal{H}$ as well as in its eigenvalues $\mathcal{E}_{i}$ and eigenfunctions $\Phi_{i}$. For distant levels, the feedback can be neglected, to a good approximation, and the non-Hermitian Hamiltonian $\mathcal{H}$ passes smoothly into the standard Hermitian Hamiltonian $H^{B}$ (for details see [6] ).

The coupling of the system to the common environment of scattering wavefunctions entails some non-trivial mathematical problems. First of all, there are singular points (EPs) in the continuum at which the influence of the environment onto the system is extremely large and which therefore influence strongly the dynamics of open quantum systems. They cause nonlinear terms in the Schrödinger equation and non-rigid phases of the wavefunctions, as shown and discussed in ref. [6] for isolated EPs. Different EPs influence each other when the ranges of their interaction overlap. Here, a clustering of EPs occurs and causes a strong mixing of the wavefunctions of all states in a finite parameter range. According to the numerical results obtained for three states, the eigenvalues of all the three eigenstates of $\mathcal{H}$ will never cross exactly in one point in a physical system. Another important feature is that the environment can put its information on an infinite number of degrees of freedom (continuum of scattering wavefunctions) at the EP into the system. This mechanism is strengthened when the system has more than two states as shown in the present paper. This mechanism is aimed at the stabilization of the system by accumulation almost the whole coupling strength between system and environment onto one state (in the one-channel 
case). EPs may cause therefore DPTs with non-analytical connected states on both sides of the DPT. This statement corresponds to the results obtained qualitatively some years ago [1] for the appearance of a phase transition in an open quantum system described by a non-Hermitian Hamilton operator.

The results shown and discussed in the present paper are obtained for open quantum systems described by a Schrödinger equation while many experimental results on DPTs are known in optics. An example is the well-known Dicke superradiance [5] and the recent studies on systems with loss and gain that started with [13 -15] and are continued in many different investigations, e.g. [16 18]. In the studies with gain and loss, the equivalence of the optical wave equation and the quantum mechanical Schrödinger equation [19 21] (which holds true at least under special conditions) is used to explain the experimental results by means of a phase transition occurring under the influence of EPs. The results of the present paper are expected therefore to be applicable also to the description of optical phenomena. Of special interest is the open question whether the Dicke superradiance [5] known since many years, is a DPT of the type discussed in the present paper. In [7], it has been shown that the transition from Autler-Townes-splitting to electromagnetically induced transparency in optics might be understood as a DPT between two very different processes taking place as a function of the Rabi frequency. Other studies on a DPT and EPs in optics are performed very recently [22, 23].

In any case, the results of the present paper will allow us, on the one hand, to receive a better understanding of the properties of open quantum systems and of DPTs. On the other hand, they will allow us to design new devices with desired properties.

[1] C. Jung, M. Müller, and I. Rotter, Phys. Rev. E 60, 114 (1999)

[2] W.D. Heiss, M. Müller and I. Rotter, Phys. Rev. E 58, 2894 (1998)

[3] T. Kato, Perturbation Theory for Linear Operators, Springer, Berlin 1966

[4] I. Rotter and J.P. Bird, A Review of Progress in the Physics of Open Quantum Systems: Theory and Experiment, Rep. Prog. Phys. 78, (2015); Shortened version arXiv:1507.08478 (July 2015) 
[5] R.H. Dicke, Phys. Rev. 93, 99 (1954)

[6] H. Eleuch and I. Rotter, Eur. Phys. J. D (2015)

[7] H. Eleuch and I. Rotter, Eur. Phys. J. D 68, 74 (2014)

[8] I.N. Bronstein, K.A. Semendjajew et al., Taschenbuch der Mathematik, Verlag Harri Deutsch, 24. Auflage (1989).

[9] I. Rotter, J. Phys. A 42, 153001 (2009)

[10] G. Demange and E.M. Graefe, J. Phys. A 45, 025303 (2012)

[11] W. Iskra, I. Rotter and F.M. Dittes, Phys. Rev. C 47, 1086 (1993)

[12] Y. Yoon, M.G. Kang, T. Morimoto, M. Kida, N. Aoki, J.L. Reno, Y. Ochiai, L. Mourokh, J. Fransson, and J.P. Bird, Phys. Rev. X 2, 021003 (2012)

[13] A. Guo, G.J. Salamo, D. Duchesne, R. Morandotti, M. Volatier-Ravat, V. Aimez, G.A. Siviloglou and D.N. Christodoulides, Phys. Rev. Lett. 103, 093902 (2009)

[14] C.E. Rüter, G. Makris, R. El-Ganainy, D.N. Christodoulides, M. Segev, and D. Kip, Nature Physics 6, 192 (2010)

[15] T. Kottos, Nature Physics 6, 166 (2010)

[16] C.M. Bender, M. Gianfreda, S.K. Özdemir, B. Peng, and L. Yang, Phys. Rev. A 88, 062111 (2013)

[17] B. Peng, S.K. Özdemir, F.C. Lei, F. Monifi, M. Gianfreda, G.L. Long, S.H. Fan, F. Nori, C.M. Bender, and L. Yang, Nature Physics 10, 394 (2014)

[18] B. Peng, S.K. Özdemir, S. Rotter, H. Yilmaz, M. Liertzer, F. Monifi, C. M. Bender, F. Nori, and L. Yang, Science 346, 328 (2014)

[19] A. Ruschhaupt, F. Delgado and J.G. Muga, J. Phys. A 38, L171 (2005);

R. El-Ganainy, K.G. Makris, D.N. Christodoulides and Z.H. Musslimani, Optics Lett. 32, $2632(2007)$

[20] K.G. Makris, R. El-Ganainy, D.N. Christodoulides and Z.H. Musslimani, Phys. Rev. Lett. 100, $103904(2008)$

[21] Z.H. Musslimani, K.G. Makris, R. El-Ganainy and D.N. Christodoulides, Phys. Rev. Lett. 100, $030402(2008)$

[22] M.H. Teimourpour, R. El-Ganainy, A. Eisfeld, A. Szameit, and D.N. Christodoulides, Phys. Rev. A 90, 053817 (2014)

[23] D. Nagy and P. Domokos, arXiv:1503.04672 (2015) 